\title{
Computer Vision Localization Based On Pseudo-Satellites
}

\author{
THESIS
}

Presented in Partial Fulfillment of the Requirements for the Degree Master of Science in the Graduate School of The Ohio State University

\author{
By \\ Kevin Robert Huggins \\ Graduate Program in Electrical and Computer Engineering
}

The Ohio State University

2009

Master's Examination Committee:

Dr. Yuan F. Zheng, Advisor

Dr. Bradley D. Clymer 


\section{Copyright by}

Kevin Robert Huggins

2009 


\begin{abstract}
Computer vision offers a unique opportunity to stand-alone navigation or use in combination with other systems. In this thesis, we propose new localization and navigation methods using computer vision techniques to detect the distance to targets and localize a platform based on the distance.

There are multiple methods of navigation such as global positioning systems (GPS) and global systems for mobile communication (GSM). However, these methods of navigation rely on receiving information from extrinsic sources. GPS requires signals from 4 satellites in order to accurately localize the receiver. However, if signals from 4 satellites cannot be obtained, which could happen in urban areas or areas with high foliage, the position of the receiver cannot be calculated accurately. We propose to use a hybrid computer vision system with GPS when one or more of the satellites are unavailable. The pseudo-satellite approach uses a network of nodes. Each node has the possibility of acting as a pseudo-satellite for another node that does not have the necessary 4 signals from satellites.
\end{abstract}

We propose an approach to localization that uses computer vision to select the most probable current location based on preregistered local knowledge and new knowledge gathered as the platform moves in the environment. This computer vision approach uses multiple techniques to sense distance using a camera mounted on the 
agent. This distance information may then be integrated with the pseudo-satellite approach for increased accuracy. The computer vision approaches range from using predetermined knowledge of object size or location to using orientation of fixed objects and vehicle motion for determining distance and orientation. The approach relies on the camera parameters calculated from the camera calibration.

In this thesis, each technique to calculate distance is tested and the results are compared with the actual distances. A program that automatically detects objects in the environment and calculated distance is compared with the other methods of calculating distance by manually detecting the object. The program performs well and gives better error rates at the tested distances to objects. 
To my mother, Darlene Huggins, and sisters, Courtney and Kasey, and Melissa Hawdon 


\section{ACKNOWLEDGMENTS}

I would like to thank my advisor, Prof. Yuan F. Zheng, for his support and guidance over the last 2 years. I thank him for taking me as a graduate student and showing me new research areas. I also thank him for the weekly meetings and pushing me to achieve great things. Without his instruction this thesis would not have been completed.

I would like to thank Prof. Bradley Clymer for being on my committee and reading through the draft of my thesis.

I would like to thank Michael McGrath for working with me on the NAECON paper last year and getting the computer vision ideas off the ground with me.

I also thank all my lab members, Naresh, Yuanwei, Junda, Yen-Lun, Liang, and Rittavee, for the help and guidance while studying to complete my degree. 


\section{VITA}

\begin{tabular}{|c|c|}
\hline June $2001 \ldots . . . . .$. & Bexley High School \\
\hline December 2007 & B.S. Electrical and Computer Engineering, \\
\hline & The Ohio State University \\
\hline 2008 to present & Graduate Teaching Associate, Department \\
\hline & of Electrical and Computer Engineering, \\
\hline & The Ohio State University \\
\hline July $2009 \ldots$. & Winner of NAECON Grand Challenge with \\
\hline & paper "Computer Vision Localization Based \\
\hline & on Pseudo-Satellites" \\
\hline
\end{tabular}

\section{FIELDS OF STUDY}

Major Field: Electrical and Computer Engineering 


\section{TABLE OF CONTENTS}

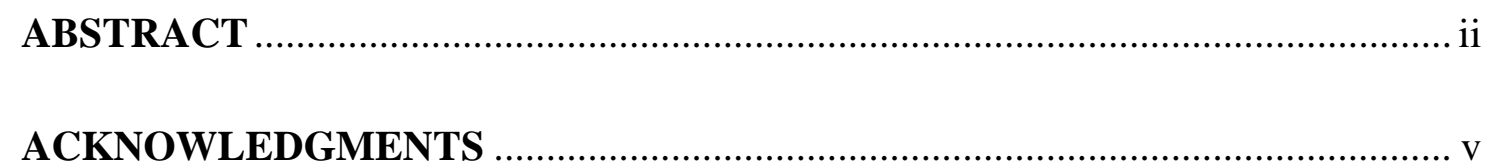

VITA

TABLE OF CONTENTS ............................................................................................ vii

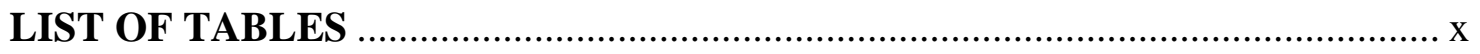

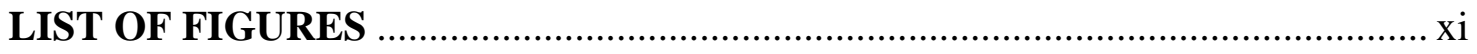

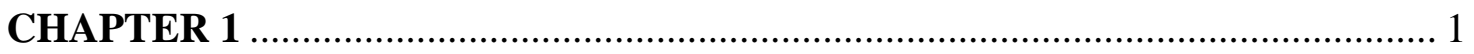

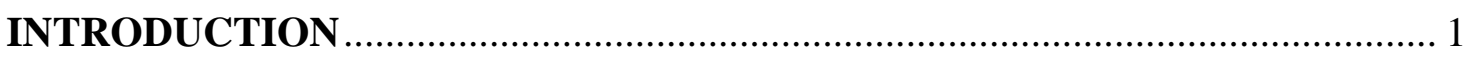

$1.1 \quad$ Global Positioning System ……………………..................................... 1

1.2 Pseudo-Satellite Approach ……………………..................................... 4

$1.3 \quad$ Proposed Approach................................................................................. 5

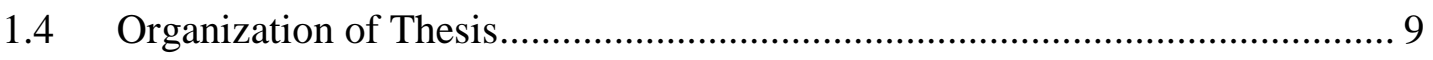

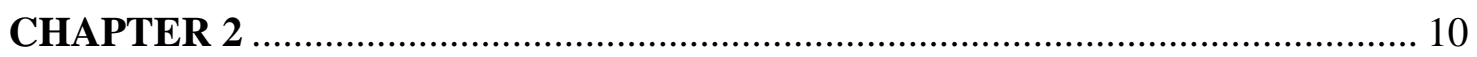

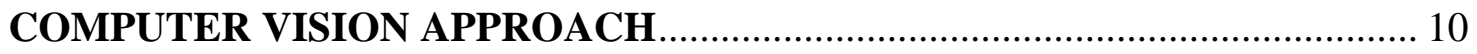

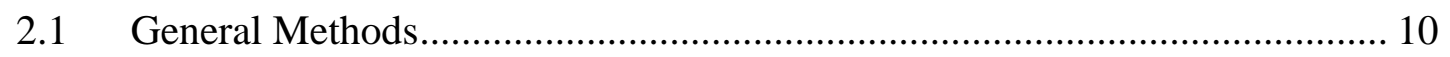




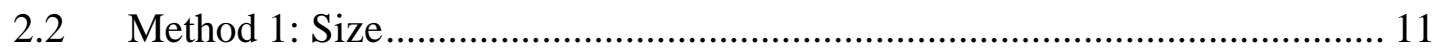

2.3 Method 2: Height and Location................................................................... 14

2.4 Method 3: Traveling Distance ……………….............................................. 15

2.5 Method 4: Indirect Traveling Distance.......................................................... 17

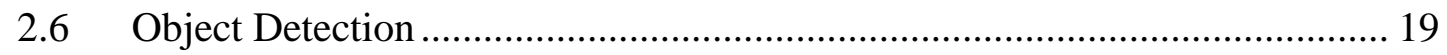

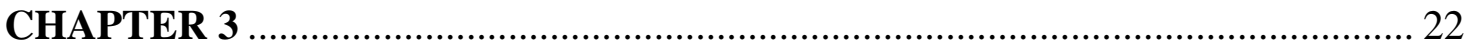

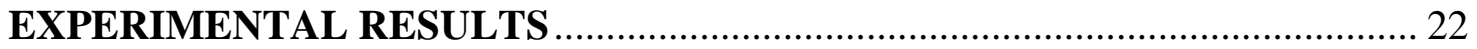

3.1 Experimental Setup.............................................................................. 22

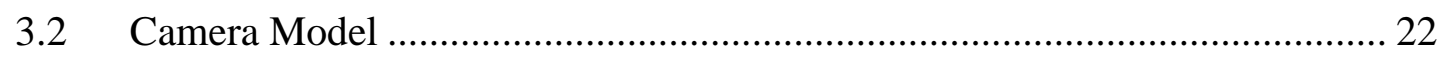

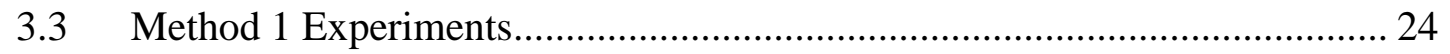

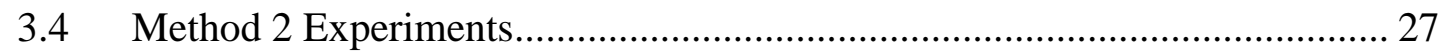

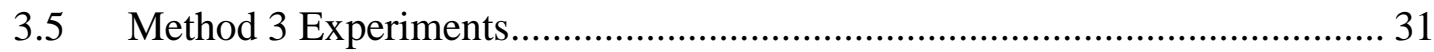

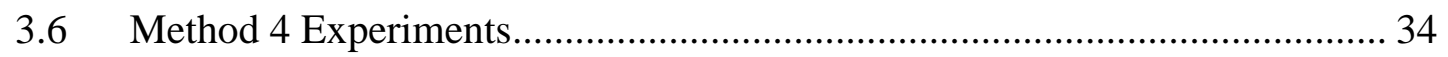

3.7 Object Extraction and Automatic Distance Calculation .................................. 37

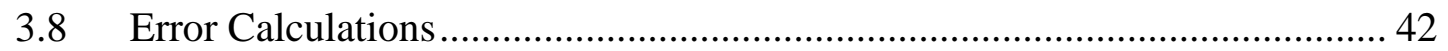

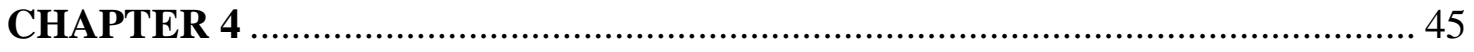

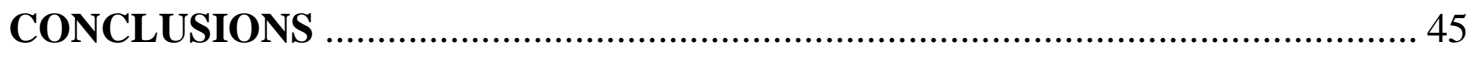

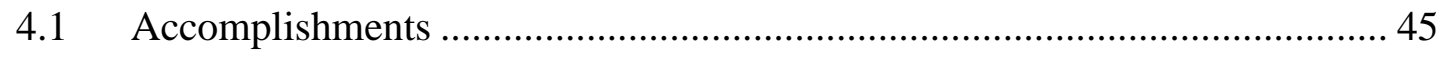

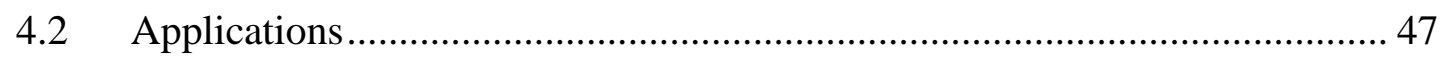

viii 


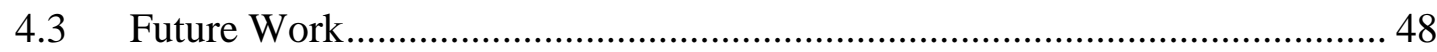

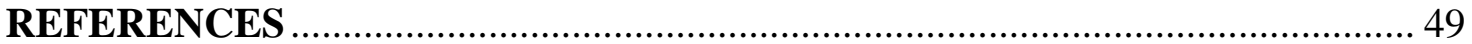




\section{LIST OF TABLES}

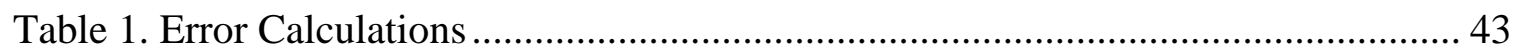




\section{LIST OF FIGURES}

Figure 1. The Pseudo-Satellite Approach ................................................................. 5

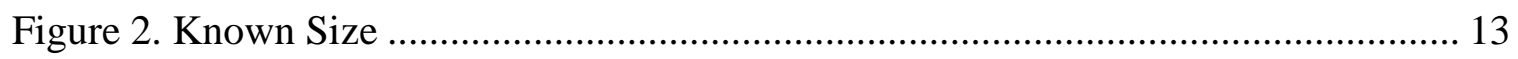

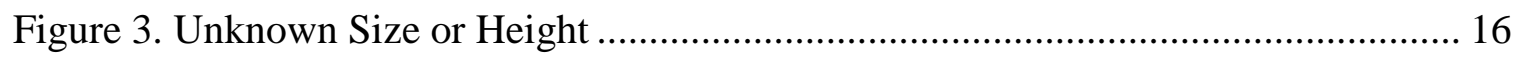

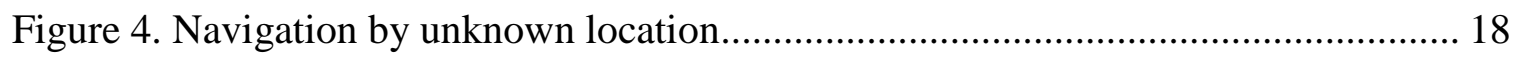

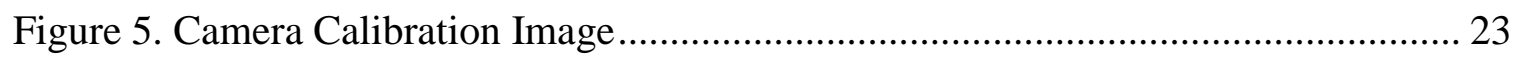

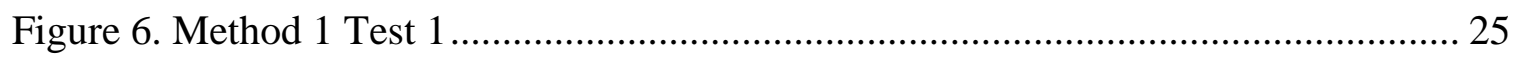

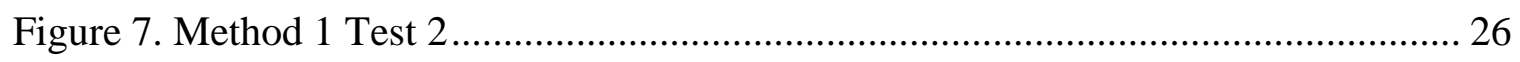

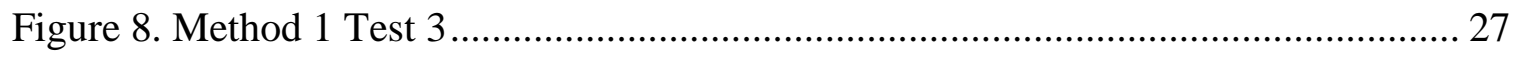

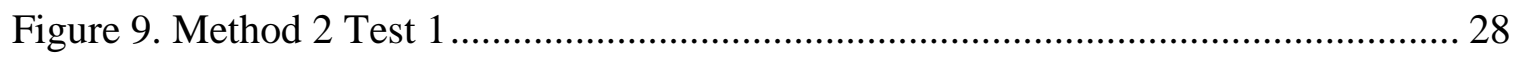

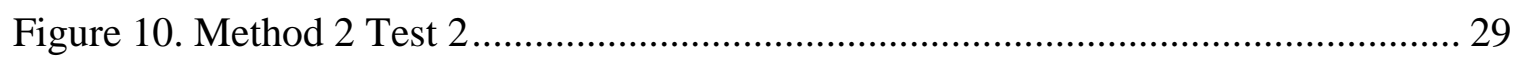

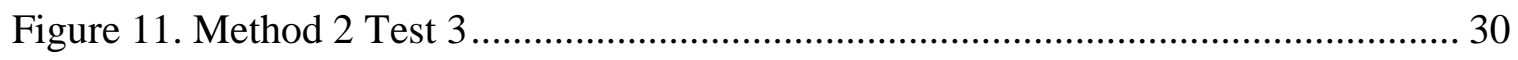

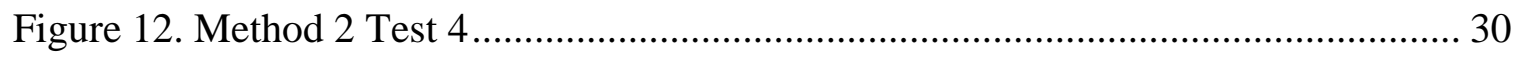

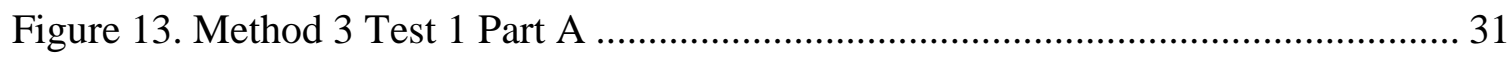

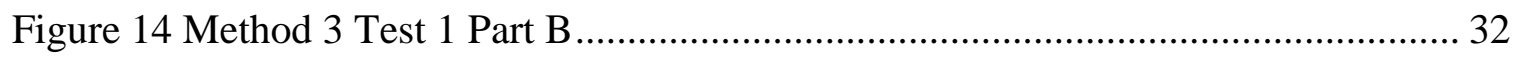

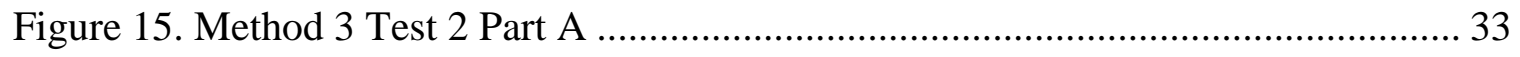

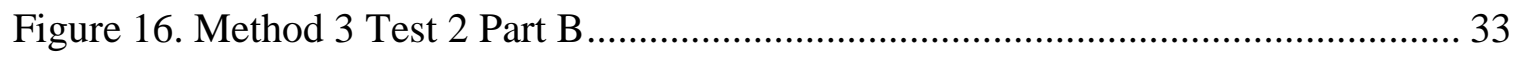

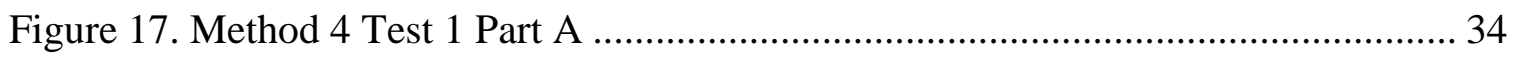

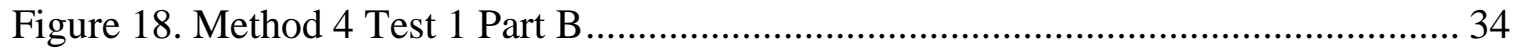




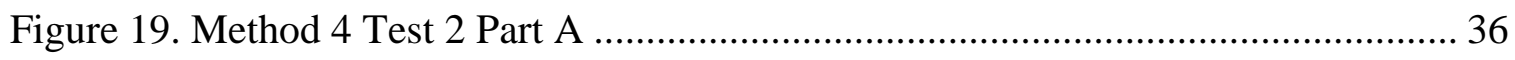

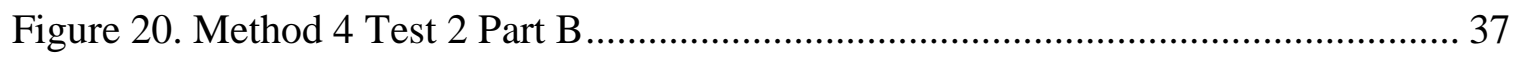

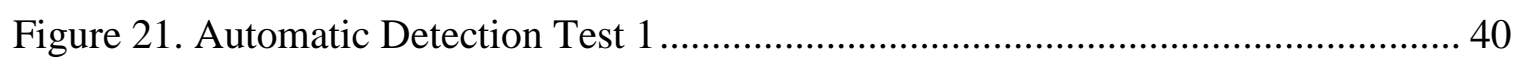

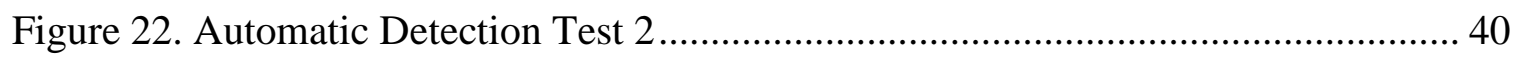

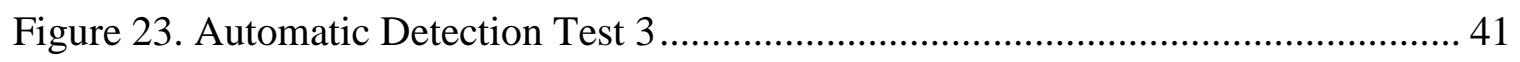

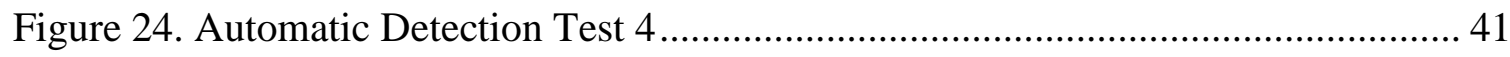

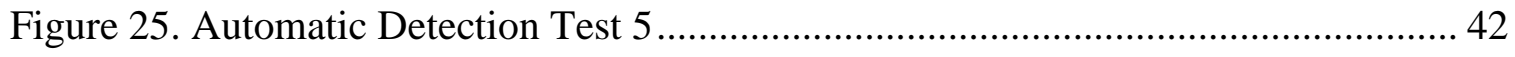




\section{CHAPTER 1}

\section{INTRODUCTION}

\subsection{Global Positioning System}

Global Positioning Systems became available to the public in the 1990's after the United States Government made the 24 orbital satellites available to consumers. Global Positioning Systems (GPS) is a way to find the location of an object on the Earth. The US military funded GPS to aid in troop logistics and navigation. GPS works by receiving radio signals from four orbital satellites; when the GPS receiver is within reach of four signals it can accurately calculate the position of the receiver [5]. The method to calculate the location is called trilateration.

Trilateration is a mathematical technique used to calculate the position of a point from three surrounding points by using the intersection of circles in $2 \mathrm{D}$ and spheres in 3D. GPS uses trilateration to calculate the speed, position, and elevation of the receiver. The GPS satellites send radio signals with information about the satellites velocity, current location, and time the signal was sent [5]. When the GPS receives the signal, it calculates the Time Difference of Arrival (TDOA) [2]. TDOA takes the time the message was sent and subtracts it from the time the message was received. 


$$
T D O A=(t r-t)
$$

where $t r$ is the time the message was received and $t$ is the time the message was sent. To calculate the distance from the receiver to the satellite, TDOA is multiplied by the speed of light.

$$
(\operatorname{tr}-t) * c=d
$$

where $c$ is the speed of light and $d$ is the distance. When a receiver calculates the distance to a satellite, the exact position of the receiver is still unknown because it could be anywhere on a sphere centered at the satellite with the calculated radial distance. When the distance to three satellites is known the intersection of the spheres is constructed to find the relative 3-space receiver coordinates. However, 4 satellites are needed to accurately calculate the position of the receiver due to the clocks on the satellites and on the receiver needing high precision and synchronization to obtain an accurate position. If the clocks are off by a microsecond, the GPS position could be off by hundreds of meters. Therefore, the fourth satellite distance is used for calibration of the clocks.

GPS has become a powerful tool, not only for military purposes, but for recreational and public purposes. GPS receivers are inexpensive and widely available to all consumers. However, the accuracy of the GPS receiver depends on the hardware that accompanies the device. Today, GPS receivers are accurate anywhere from 3-100 meters depending on the hardware and where the receiver is being operated.

The receiver always needs four visible satellites to calculate its position. Inability to obtain signals from four satellites is a problem that occurs in urban areas where large 
buildings and obstructions block radio signals. In heavily wooded areas, dense foliage creates a canopy that may prevent a GPS receiver from reading four satellite signals. There have been many different approaches to solve this problem. When the current elevation is known then only three satellite signals need to be visible. Another approach would be to use other GPS receivers as pseudo-satellites. Alternatively, the pseudosatellite approach may use computer vision techniques as discussed further in section 1.2. Another form of localization and positioning that could be used as a substitute when GPS is unavailable is single or multi-channel cellular phone localization based on signal strength. Mobile phones position can be found even when the phone is not making a call. As long as the cell phone is turned on it sends out a roaming signal. This roaming signal tries to find the nearest base station. When it finds the nearest base station the signal strength is determined. A method known as multilateration is used to find the position of the mobile phone. Multilateration is similar to trilateration. A mobile phone will try to find the nearest base station, but at the same time it can also communicate with other base stations. If three base stations know the signal strength to the mobile phone then the position of the phone may be calculated. Similar to GPS, TDOA is used to calculate the distance from the phone to the base station. However, three base stations cannot always be contacted such as in rural areas. When this occurs only one distance can be calculated and the exact position cannot be discovered. To compensate for this problem, GPS is being installed on mobile phones to form a hybrid solution to the limitations of cellular, network-based localization [4]. 


\subsection{Pseudo-Satellite Approach}

The GPS receivers may fail due to various environments preventing position calculation. A Pseudo-Satellite approach is proposed to solve this problem. This approach is based on a network of receivers, or nodes, where some of the nodes can be used as pseudo-satellites. The pseudo-satellites would be available to the other nodes in the network that do not know their position. GPS would be used to calculate the position of nodes that have 4 satellites visible. Then the other nodes that do not have 4 satellites visible can use the position of the pseudo-satellites to find their position. Using this approach the nodes have to be able to communicate with one another [3]. To further illustrate this approach refer to Fig. 1.

Fig. 1 features four satellites visible from Node1; therefore, its position $p_{1}(k)$ can be calculated, where $k$ indicates the time. However, Node 2 can only receive three satellite signals, and therefore, its position cannot be accurately determined. But if the range $r_{1,2}(k)$ between nodes 1 and 2 is known, the position of Node $2 \mathrm{p}_{2}(k)$ can be estimated. By making use of $p_{1}(k)$ and $r_{1,2}(k)$ Node 1 plays the role of the fourth satellite to Node 2. As shown in Fig. 1, Node 1 receives four GPS satellite signals (blue lines), Node 2 receives three GPS satellite signals and one pseudo-satellite signal (red lines), Node 3 receives two GPS satellite signals and two pseudo-satellite signals (green lines), and Node 4 receives one GPS satellite signal and three pseudo-satellite signals (brown lines). All the nodes in the network receive four satellite or pseudo-satellite signals. Thus all their positions may be determined [3]. 


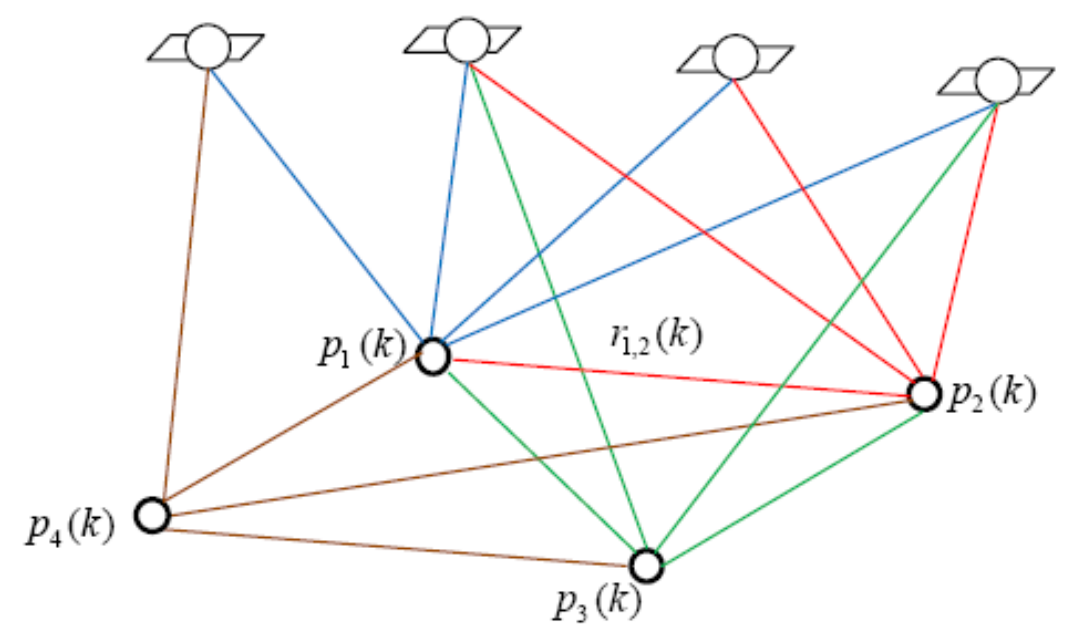

Figure 1. The Pseudo-Satellite Approach

\subsection{Proposed Approach}

We propose to use computer vision techniques to search for visible landmarks. The landmarks would serve as pseudo-satellites and can range from known landmarks outdoors, such as street signs or buildings, to recognizable objects indoors such as plaques for room numbers or decorations on the wall. One advantage to using computer vision is flexibility in search for the landmark. Multiple objects could serve as a landmark or pseudo-satellite, compared to GPS receivers, which must use signals from satellites as the landmark. The same applies to cell phones, because a cell phone uses a base station as the landmark. The computer vision approach offers a more robust search for the pseudo-satellite. If one landmark is not visible to the camera, then the platform could turn in place and search for another landmark known in the area. However, if a satellite is not visible to a GPS receiver, the platform would have to travel in order to find 
the signal. In areas of dense foliage the distance traveled could be miles, or in urban areas the distance could be multiple city blocks.

We propose to use a single camera to calculate the range to the visible landmarks. The range calculation is based on prior knowledge of the landmark. We have developed four different scenarios for calculating the distance based on the size of the landmark, the height and location of the landmark, the angle from the camera's horizon to the landmark combined with a distance traveled, and two angles from the camera's center to the landmark combined with a distance traveled. As previously discussed; GPS uses TDOA to determine the range from the receiver to the satellite. When a GPS receiver does not have four satellites available, the position cannot be calculated. The conventional approach to fix this problem is to use a fixed radio station as a pseudo-satellite [2]. The difficulty is that the TDOA must be accurate; otherwise, the distance errors will be huge due to the light speed of the microwave. TDOA requires that the clocks on the receiver and satellite be synchronized in order to determine an accurate range measurement. If the synchronization is off between the two, the error in the distance calculation could be very large and this is problematic. For localization indoors, GPS is not reliable because the strength of the signal is weak and it cannot penetrate thick walls. Therefore, the distance measurement is not accurate. In contrast, computer vision does not rely on TDOA to determine the range and it is not affected by signal strength. It relies on an image and the size and location of the object or landmark. Our distance calculation approach is very accurate and overcomes the problems of GPS and cell phone localization. 
The computer vision approach would be ideal for indoor navigation and localization where GPS and other localization methods do not perform well. Kim and Jun developed a computer vision method for indoor localization. They use a wearable PC and a mounted camera on a person in order to determine location. The PC sends images to desktop PCs where the image sequences are processed to determine location [8]. This method uses an image database to find a target and determine the location. Prior knowledge of the environment is essential for this method to succeed. Multiple images of the environment must be stored in order to match the image sequences coming in from the camera. Park, Rasheed, and Beak developed an indoor navigation system that detects arrow signs that direct a mobile robot on which way to turn [7]. This approach only detects arrow signs and does not localize the position of the robot; it is solely used for navigation.

Our proposed computer vision approach could be used for navigation and localization. The prior knowledge that our computer vision approach requires is the location of the landmark. As previously discussed, the landmark is a pseudo-satellite, so the landmark already knows its location and if the range to the pseudo-satellite can be calculated then the location of the platform can be determined. The only required image processing is being able to detect the landmark within the image. We propose to use a simple landmark for indoor navigation that is easily detectable and is printed on a standard 8.5 inch by 11 inch sheet of paper. Knowing the size of the landmark provides enough prior knowledge to determine the distance to the target in one of the proposed distance calculation methods. The proposed computer vision approach uses a laptop and 
a webcam as the platform to determine distance and the position from the landmark. This approach does not use multiple PCs, which adds to the cost of the system. Also, this approach does not use an image database which would require a lot of computing time.

Our proposed computer vision approach could also be used as a distance calculation with a camera. As long an object of known size or location is in the field of view of the camera the distance to the object can be found. Hsu, Lu, and Chin developed a distance calculation using triangular relationships that could be implemented with CCD cameras [9]. Their method called for selecting two arbitrary points in an image then moving the camera and taking another picture and finding the same points in the image. Then using the pixel difference between the selected points, and the actual distance moved by the camera the range was calculated. However, this is their only method to calculate the distance using a camera. There are many constraints to this approach. One constraint is that the arbitrary selected points would have to remain still in the two images or the distance measurement would be off. Also, the distance moved would have to be standard if this approach were implemented on CCD cameras. In contrast, our approach covers four methods to determine the range to the object. In two of our methods the camera does not need to move to acquire a range measurement.

The proposed computer vision approach may be used for navigation and localization or simply as a distance calculation with a camera. It does not rely on TDOA or signal strength to determine the range to the landmark. Also, the proposed approach overcomes the shortcomings of other proposed computer vision localization methods by 
not requiring much computing power or multiple devices. The prior knowledge needed for the proposed method is minimal as compared to other methods.

\subsection{Organization of Thesis}

Chapter 2 discusses the theory for the four methods to determine distance using a camera and an object of known size and location. Chapter 3 provides the experimental setup and results for testing the various computer vision techniques. We conclude our work in Chapter 4 by discussing what has been accomplished, what applications this theory can be used for, and what will be the future work for this topic. 


\section{CHAPTER 2}

\section{COMPUTER VISION APPROACH}

Computer vision based estimation of location enhances the pseudo-satellite approach by providing localized opportunities based on prior knowledge of the size, location, and/or orientation of objects within view of the camera. By utilizing the known camera parameters and field of view the platform's position and orientation may be calculated.

\subsection{General Methods}

Whereas with GPS-based approaches the vehicle would need to move to discern orientation, computer vision enhances orientation if turning in place or if motion history is lost but a recognized object is within the field of view. Objects that are visible from all sides but textured uniquely to each vantage point offer the most flexibility. An example of such an object would be a sphere divided into a northern and southern hemisphere with n-latitude lines such that each section receives a different color.

Objects not visible from all sides offer less utility in determining location but it is not necessary for position-unique texture when the field of view of each object only has one intersection. When using satellites and objects visible from all sides without 
position-unique textures, there may be two intersections of their radii and therefore two possible positions and orientations.

Orientation may be inferred based on the type of object. If the object is one-sided and is in a known location, viewing the object straight-on gives both distance and orientation. If a flat object of known size is positioned vertically and approached from a side angle, the height of the object still allows the distance from the object to be found, while the compression of the object's horizontal pixels gives the angle of the camera from normal. If the object has a unique view from any angle, knowing the distance and view will provide location and orientation. If the object appears the same from all angles, at least two discernable objects of known locations will be needed in the field of view to determine orientation and the size of at least one object will be needed for location.

\subsection{Method 1: Size}

Knowledge of the camera model is used to determine distance of objects with known size. Our approach approximates images on a per-pixel level that is dependent on the camera geometry. Each pixel represents an arc length dependent on the focal length and geometry of the camera lens. Assuming the sensor is a flat surface, the per-pixel arc length may be approximated by taking the camera's total horizontal and vertical fields of view and dividing by the resolution of the camera. The field of view of a camera is the angular extent of the world as seen by the camera. Each camera has its own vertical and horizontal field of view. The field of view will not change if the focal length of the camera stays constant. One approach to calculating the field of view can be accomplished by taking an image from a known distance and having an object of a 
known size in the optical center of the image. From the known parameters a right triangle can be formed with the distance from the camera, the width in inches of half of the image frame, and the angle from the camera. The angle is half the horizontal field of view and the following equations show how to calculate it, and they can be extended to determine the vertical field of view.

$$
\frac{w \text { inches }}{z \text { pixels }}=g \frac{\text { inches }}{\text { pixel }}
$$

Frame width pixels $* g \frac{\text { inches }}{\text { pixel }}=x$ inches

$$
\tan ^{-1}\left(\frac{\frac{x \text { inches }}{2}}{d}\right)=\alpha^{\circ}
$$

In the equations $w$ is the real world width of the object, $z$ is the number of pixels that the object occupies in the frame, $x$ is the number of inches that the width of the frame occupied in the real world, $d$ is the distance to the object, and $\alpha$ is half of the horizontal field of view. Once the horizontal field of view is known, the arc length of each pixel can be calculated by

$$
\frac{\text { Field of View degrees }}{\text { Frame Width pixels }}=y \frac{\text { degrees }}{\text { pixel }}
$$

This too, can be extended to the vertical field of view and the degrees per pixel can be determined for each pixel vertically.

Knowing the horizontal and vertical arc length of each pixel in combination with prior knowledge of an object's real-world height or width allows the distance to the 
object to be determined. The calculations are similar to finding the field of view, but in this case the distance is unknown. Therefore, we can use the calculated field of view and solve for the distance to the camera by using the following equations

$$
\begin{aligned}
& \qquad \frac{w \text { inches }}{z \text { pixels }}=g \frac{\text { inches }}{\text { pixel }} \\
& \text { Frame width pixels } * g \frac{\text { inches }}{\text { pixel }}=x \text { inches } \\
& d=\left(\frac{\frac{x \text { inches }}{2}}{\tan (\alpha)}\right)
\end{aligned}
$$

where $d$ is the distance, $\alpha$ is half the horizontal or vertical field of view and is the angle represented in Fig. 2. $w$ is the real world object width, $z$ is the number of pixels that the object occupied in the frame, and $x$ is the number of inches that the entire frame occupies in the real world. The right triangle in Fig. 2 represents the horizontal viewpoint from the camera. The right triangle consists of half of the horizontal field of view angle, the distance from the camera to the object, and half of the frame width represented in inches.

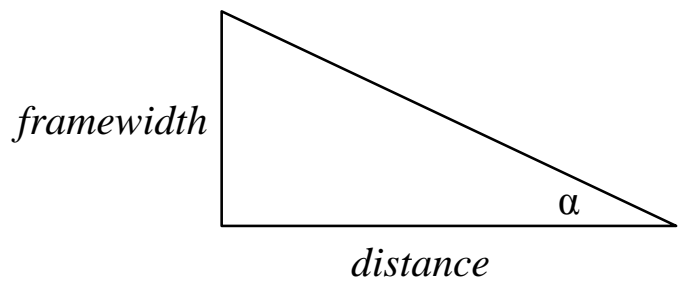

Figure 2. Known Size

The one constraint on Method 1 is that the object must be placed in the optical center of the image. This allows the right triangle to be formed and the distance to be 
calculated. Assuming that the object is one-dimensional, like a sheet of paper, and the object can be seen in the field of view of the camera the location of the platform can be determined by the distance. The orientation can also be discovered by the distance calculation. Since, the camera can see the object, it can be inferred that the platform must be in front of the image a calculated distance away.

\subsection{Method 2: Height and Location}

Method 2 involves knowing the height and location of the target. If the size of the target is not large enough to provide an accurate distance measurement, then another approach must be formulated to calculate the distance to the object. As mentioned if the height of an object is known, then a triangle can be formed between the optical center, the center of the target, and the camera. The distance to the target may be calculated in a similar fashion to the previous method. A constraint on this method is the target must be directly above the optical center of the image. This ensures that a right triangle can be formed. Using the prior knowledge of the arc length in the vertical direction an angle can be found based on how many pixels between the optical center and the center of the target, as shown in the following equation

$$
\alpha=a * b \frac{\text { degrees }}{\text { pixel }}
$$

where $\alpha$ is equal to the angle between the optical center and the center of the target, $a$ is equal to the number of pixels between the optical center and the center of the target, and $b$ is the vertical arc length per pixel. Finally, the distance can be calculated based on the following equation 


$$
\text { distance }=\frac{h \text { inches }}{\tan (\alpha)}
$$

where $h$ is the height of the target from the optical center and $\alpha$ is the angle previously calculated.

With the distance to the object, we have reduced the number of possible locations to a circle around the target of radius distance. This is similar to receiving a distance measurement via one GPS satellite. If the target were three dimensional, and the location could not be determined by the single target, another target would have to be present in the field of view of the camera to localize the position of the platform. However, the location is constrained by knowing the object is a two dimensional sheet of paper. Therefore, the location and orientation can be found by the location of the target in the field of view of the camera.

\subsection{Method 3: Traveling Distance}

The distance to a target can be determined even if the height of the target is unknown, as long as the target is in the field of view of the camera. This is done by acquiring the target directly above or below the optical center of the camera, calculating the angle to the target, moving directly towards the target, and calculating the angle again. Using the knowledge of the camera geometry, and by knowing the distance traveled by the platform, the distance to the target can be determined. The distance traveled by the platform could be calculated automatically, but if that is not available, a second target with known parameters could be used to calculate the distance traveled. 
Using the distance moved $m$ and the two vertical angles, as seen in Fig. 3, the following equation gives the total distance to the object

$$
d=\frac{\sin (\alpha)}{\sin (\beta-\alpha)} \cdot m
$$

where $d$ is equal to the distance. Also, the ground distance or the distance to the optical center can be calculated by

$$
g d=\frac{\sin (\alpha)}{\sin (\beta-\alpha)} \cdot m \cdot \cos (\beta)
$$

where $g d$ is the ground distance and the angles are represented in Fig. 3.

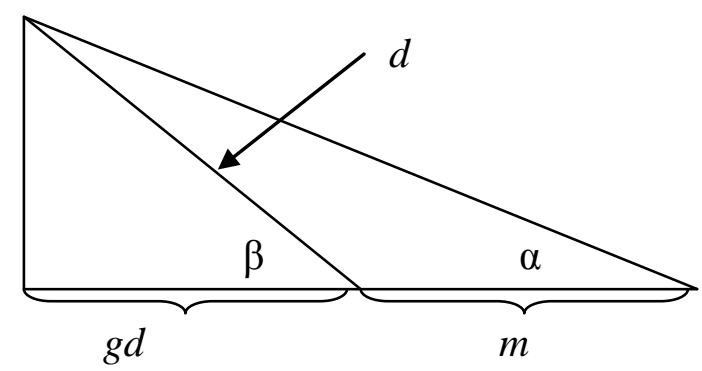

Figure 3. Unknown Size or Height

Thus using the angles and movement offers an alternative to prior knowledge of size or height. The location and orientation of the platform are determined by the targets location in the field of view. 


\subsection{Method 4: Indirect Traveling Distance}

If moving directly toward a target of unknown height but known location is not possible, Method 3 may be extended to find the distance to a target. This is done in a three stage process that consists of finding the distance along the $\mathrm{x}$ axis, finding the distance along the y axis and finally forming a final triangle to the target. This can be seen in Fig 4, where the red dot represents the target, the blue triangle represents the triangle formed along the $\mathrm{x}$ axis, the pink triangle represents the triangle formed on the $\mathrm{y}$ axis, and the black triangle represents the triangle formed with the target, the camera distance along the $\mathrm{x}$ axis, and the distance to the target. The algorithm to compute the distance follows:

Step 1. Form two triangles, one along the $\mathrm{x}$ axis and one along the $\mathrm{y}$ axis

Step 2. Calculate horizontal and vertical angles from the two triangles

Step 3. Move a distance towards the optical center

Step 4. Form the same two triangles from the new distance

Step 5. Calculate horizontal and vertical angles again

Step 6. Calculate the ground distance to the target, represented by the hypotenuse of the blue triangle

Step 7. Calculate the height of the target from the ground using the pink triangle

Step 8. Form the final triangle, using the calculated ground distance and calculated height, and calculate the distance to the target 
Using the calculated distance and the fact that the target is in the field of view of the camera the platforms position can be found.

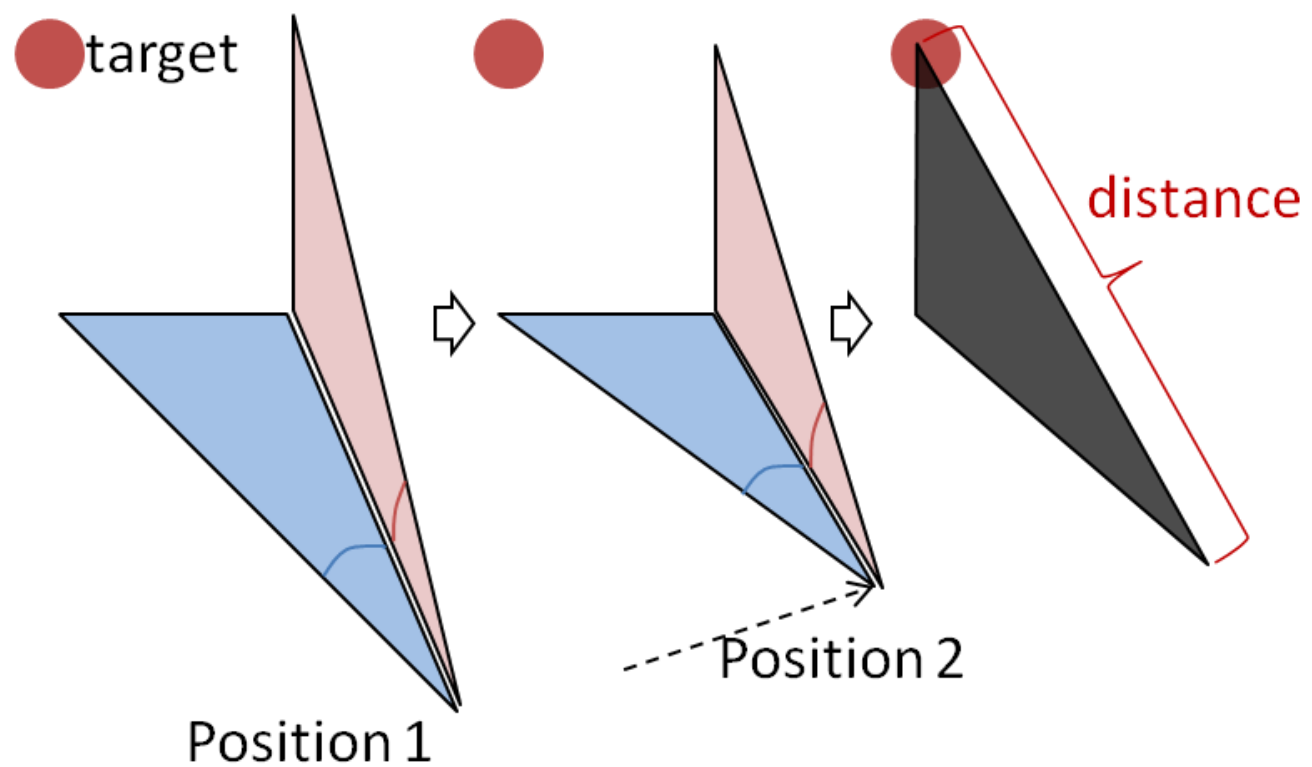

Figure 4. Navigation by unknown location 


\subsection{Object Detection}

The four methods to determine the range must have a way to detect the target. Computer vision offers multiple approaches to object detection. The first step in detecting the object is to have prior knowledge of the target. The prior knowledge serves as a model for the detection in incoming images. Recognizing key features such as color, shape, and texture of the target helps build a system that can automatically detect wanted objects in the image.

Segmentation can be used to detect objects. One form of segmentation involves background subtraction. This method takes images of an area in order to establish a background. Then when a new object appears in the image space the background images are subtracted from the new image and the new object or objects are detected [6], [11]. This detection approach relies on the lighting of the background be very similar to the lighting of the new image. When the lighting has changed, and the subtraction has been processed the algorithm could determine that the new image is entirely different from the background. This approach only detects if a new object is in the frame. However, this approach does not determine if the new object is the object that needs to be recognized. Other approaches of segmentation would be comparing color histograms or comparing edges in the images and finding the differences [6], [10].

Segmentation is often used as a starting point for detection algorithms. Park, Rasheed, and Beak used segmentation in combination with labeling, using thresholds to convert to binary images, and noise removal to detect an arrow sign in a frame [7]. In [8] the use of pattern recognition along with segmentation was used to detect a marker with a 
distinct black and white square pattern in order to determine location. For the proposed computer vision approach we selected an object that is easily recognizable with strong color and shape features. The target is a block-O picture printed on a standard 8.5 by 11 inch sheet of paper. The block-O has an octagon shape and has two main colors, red and white. In order to calculate the distance to the object, the algorithm must detect the object and detect the edges of the object.

The first step is to create a model of the object. The key features of the block-O are the small white colored octagon inside a larger red octagon. The color white has RGB values that are all the same and should equal 255 . Therefore, the white color can be detected by comparing each pixel in the frame to the color white. Next, the color red has an RGB value of $\mathrm{R}$ equal to $255, \mathrm{G}$ equal to 0 , and $\mathrm{B}$ equal to 0 . The color map of the block-O has been determined. The next step would be to use the shape of the object and determine where these colors are found. The color white is always in the center of the object and the color red is equidistant from the center of the target. Once the white color is detected then the algorithm should detect the color red a predefined distance from the center in both directions.

The second step in the algorithm would be to detect the edges of the printed sheet of paper. The block-O does not occupy the entire page it is printed on. Therefore, the color of paper around the block-O is white also. Once the object is detected the edges can be determined by searching outward from the block-O detecting the color white. When the color white is no longer found the edge of the target is determined to be in the last position where white was detected. 
Various approaches were considered in determining the algorithm to detect the object. The background subtraction algorithm could be used to detect the object if multiple images of the background were first taken. However, the computation speed to run this algorithm would be very high. It was determined that the target could be placed in multiple areas, so one background could not be used to detect the object. On the other hand, the block-O has very sharp edges and as long as the printed sheet of paper is not placed on a white wall, the edges are easily found. An edge detection algorithm converting the image to a binary image where the edges are represented with ones would be very useful. The shape of the edges could be used to determine if the block-O was detected. This could be done by labeling the connected edges. However, the processing power to implement this algorithm was too great. Therefore, the algorithm selected was based on finding the two colors, detecting the shape, and then detecting the edges. 


\section{CHAPTER 3}

\section{EXPERIMENTAL RESULTS}

\subsection{Experimental Setup}

The following experimental results were calculated using a Logitech Orbit AF webcam. The targets that the distance is being calculated to are the block-O pictures in the figures. The block-O picture was printed on an $8.5 \times 11$ inch sheet of paper. All of the images were generated using the OpenCV software with Microsoft Visual Studio 2007. The actual distance to the target was discovered by two people manually using a tape measure.

\subsection{Camera Model}

The first step in the experiments was to calibrate the camera and develop a model for the camera based on the block-O target. Using the OpenCV software a $\mathrm{T}$ was made at the middle of the frame to represent the horizon of the camera. The size for each image was $320 \times 240$ pixels. Therefore, the $\mathrm{T}$ at the middle of the images would be at pixel location 160 in the $\mathrm{x}$ direction and 120 in the $\mathrm{y}$ direction. In order to calibrate the camera the target was positioned in the middle of the frame so the $\mathrm{T}$ intersected the middle of the target, which can be seen in Fig. 5 . 


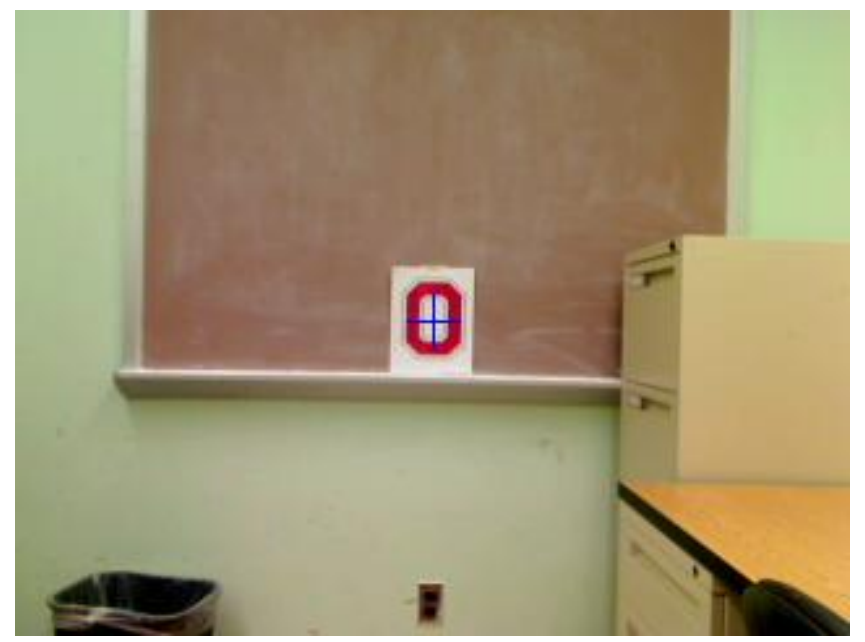

Figure 5. Camera Calibration Image

In Fig. 5 the distance from the camera to the target was measured to be 74 inches. Knowing the distance to the target, the real world size of the target, and the target being positioned in the middle of the frame the horizontal and vertical fields of view can be calculated. Using MATLAB the edges of the target were discovered and the pixel width of the target was calculated to be 30 pixels, and the vertical height of the target was 39 pixels. The horizontal field of view was calculated to be $62.98^{\circ}$ based on the following calculations.

$$
\begin{gathered}
\frac{8.5 \text { inches }}{30 \text { pixels }}=0.283 \frac{\text { inches }}{\text { pixel }} \\
320 \text { pixels } \cdot 0.283 \frac{\text { inches }}{\text { pixel }}=90.67 \text { inches } \\
\frac{90.67 \text { inches }}{2}=45.33 \text { inches }
\end{gathered}
$$




$$
\tan ^{-1}\left(\frac{45.33 \text { inches }}{74 \text { inches }}\right)=31.4903^{\circ} \cdot 2=62.98^{\circ}
$$

And the vertical field of view was $49.16^{\circ}$ and was calculated the same as the horizontal field of view. The field of view can be used to determine the arc length per pixel and this can be calculated for both directions by the following

$$
\begin{aligned}
& \frac{62.98^{\circ}}{320 \text { pixels }}=0.20 \frac{\text { degrees }}{\text { pixel }} \text { horizontal } \\
& \frac{49.16^{\circ}}{240 \text { pixels }}=0.21 \frac{\text { degrees }}{\text { pixel }} \text { vertical }
\end{aligned}
$$

The field of view calculations and the arc length will be used throughout the experiments.

\subsection{Method 1 Experiments}

The distance calculation for method 1 can be determined because the target is placed in the optical center of the image and the size of the target is known. Both of these constraints are met with the following examples because the $\mathrm{T}$ was used to position the target and the size of the target is already known to be 8.5 inches by 11 inches since it is printed on a standard sheet of paper. Also, since the field of view of the camera has already been determined the distance to the target can be calculated. Figure 6 shows the first test image for this distance calculation. 


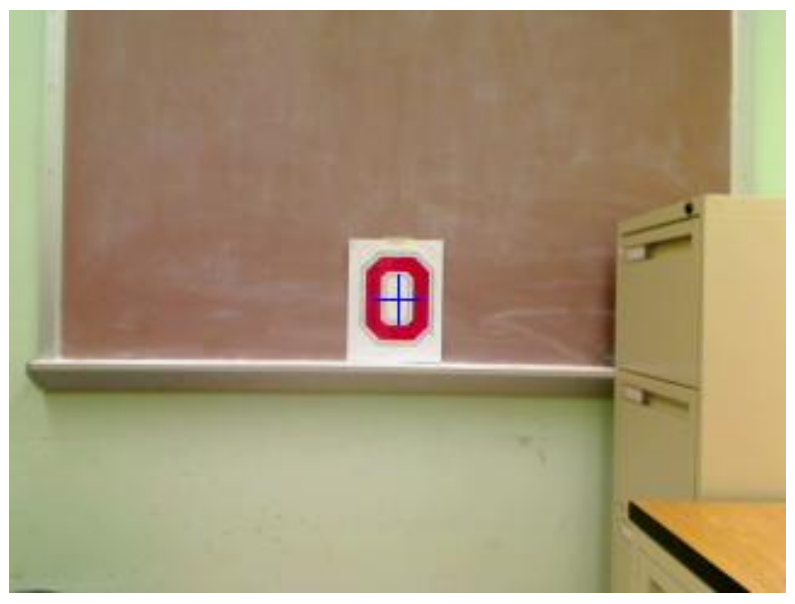

Figure 6. Method 1 Test 1

The first step to find the distance is to find the edges of the target. This was done using the edge detection function in MATLAB and manually finding the pixel locations of each corner of the target. For this image, the target occupied 37 pixels in the $\mathrm{x}$ direction and 49 pixels in the y direction. In order to calculate the distance to the target the inches per pixel were calculated by taking the size of the target in both directions and dividing it by the number of pixels occupied by the target.

$$
\begin{aligned}
& \frac{8.5 \text { inches }}{37 \text { pixels }}=0.23 \frac{\text { inches }}{\text { pixel }} \text { horizontal } \\
& \frac{11 \text { inches }}{49 \text { pixels }}=0.23 \frac{\text { inches }}{\text { pixel }} \text { vertical }
\end{aligned}
$$

Next, the total area covered by the frame in both directions was determined by multiplying the number of pixels in the $\mathrm{x}$ direction by the already calculated inches per pixel and doing the same in the y direction. After performing this calculation it is discovered that the frame covers 73.51 inches in the $\mathrm{x}$ direction and 53.88 inches in the $\mathrm{y}$ 
direction. Finally, a right triangle can be formed using one quadrant of the area covered in the $\mathrm{x}$ or the $\mathrm{y}$ direction. The angle used in the right triangle is half of the horizontal or vertical field of view. The length of one edge of the triangle is half of the area that the frame covers. The distance to the camera can be calculated by only using one direction, but calculating the distance using the information of both directions and then taking the average reduces the variance. The average distance to the target in Fig. 6 was determined to be 59.5 inches.

Two more experiments were performed using this method, but from different distances. The targets location did not change, but the position of the camera did. These scenarios can be seen in Fig. 7 and Fig. 8. The same calculations were performed and the average distance to the target in Fig. 7 was 79.73 inches. The distance calculations were performed on Fig. 8 and the average distance was 134.97 inches.

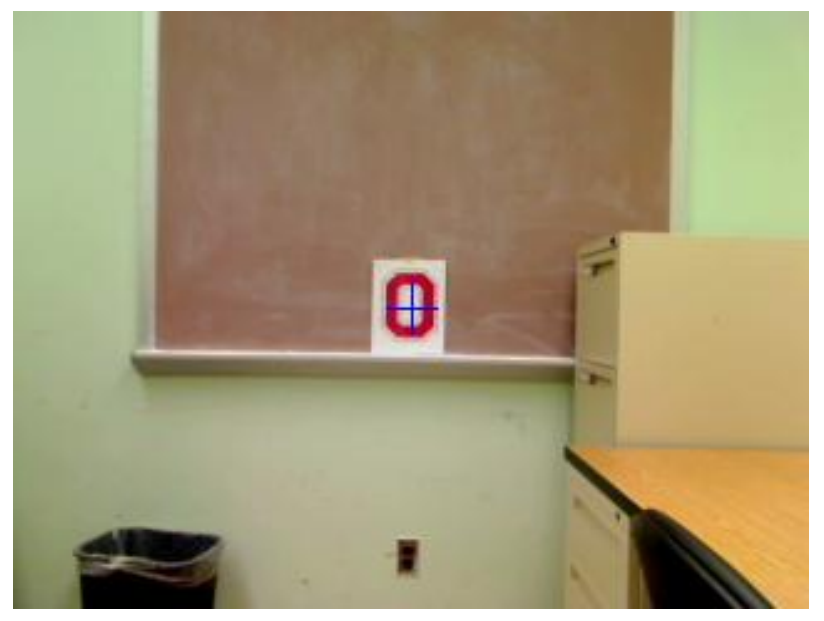

Figure 7. Method 1 Test 2 


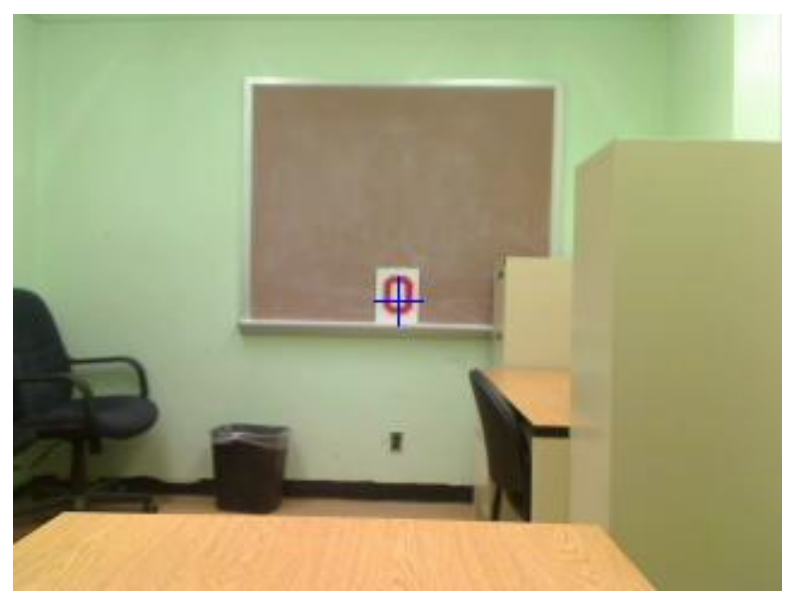

Figure 8. Method 1 Test 3

\subsection{Method 2 Experiments}

This method involves knowing the height of the object and being able to calculate the angle from the optical center of the camera to the object. One constraint is that the target must be placed directly above the optical center of the camera and on the same plane. The arc length per pixel was already calculated by calibrating the camera. Therefore, the angle from the optical center to the center of the target can be determined by how many pixels are between the two. In this experiment, the height was measured from the optical center to the center of the target using a tape measure. The optical center was marked on the wall so a distance could be measured from that point. The height was determined to be 28 inches. The first experiment using this method can be seen in Fig. 9 . 


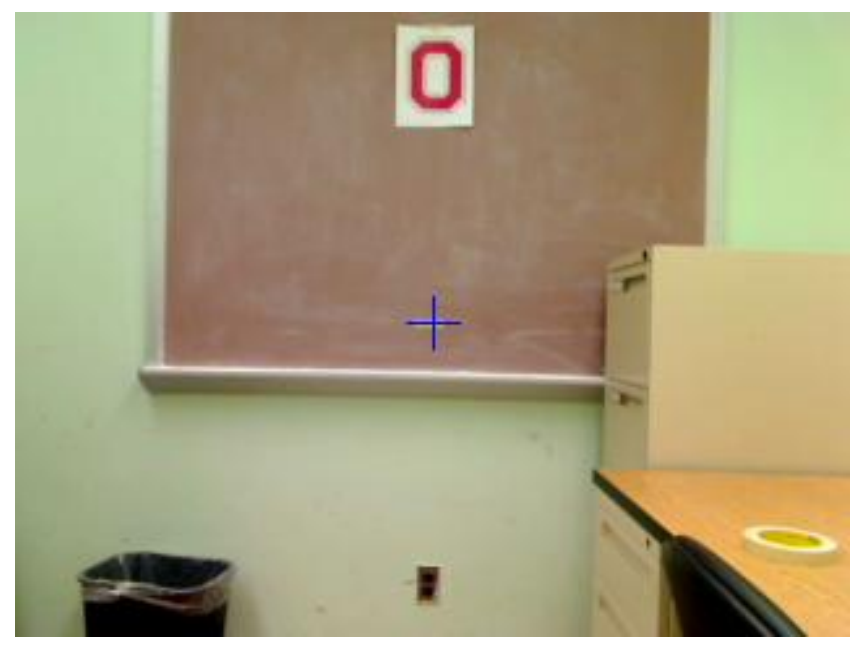

Figure 9. Method 2 Test 1

The distance to the top target was calculated by first finding the number of pixels in the y direction from the optical center to the center of the top target. The number of pixels was found by manually selecting the center of the target in Fig. 10 and finding the difference, which was 95 pixels. Next, the angle could be determined by using the arc length per pixel in the y direction.

$$
\theta=95 \text { pixels } \cdot 0.21 \frac{\text { degrees }}{\text { pixel }}=19.46^{\circ}
$$

Next, a right triangle could be drawn from the camera to the optical center up to the target. Using this right triangle the distance from the camera to the optical center was calculated by using the following formula.

$$
\text { distance }=\frac{28 \text { inches }}{\tan \left(19.46^{\circ}\right)}=79.25 \text { inches }
$$

And the distance from the camera to the center of the target was be calculated by the following formula. 


$$
\text { distance }=\frac{28 \text { inches }}{\sin \left(19.46^{\circ}\right)}=84.05 \text { inches }
$$

An additional experiment was performed using this method with the target at the same height, but the camera at a different distance from the target, which can be seen in Fig. 10. Once again, the number of pixels from the optical center to the target was found to be 76 pixels. And the angle from the camera to the target was calculated to be $15.57^{\circ}$. Finally the distances to the optical center and to the target were 100.51 inches and 104.34 inches respectively.

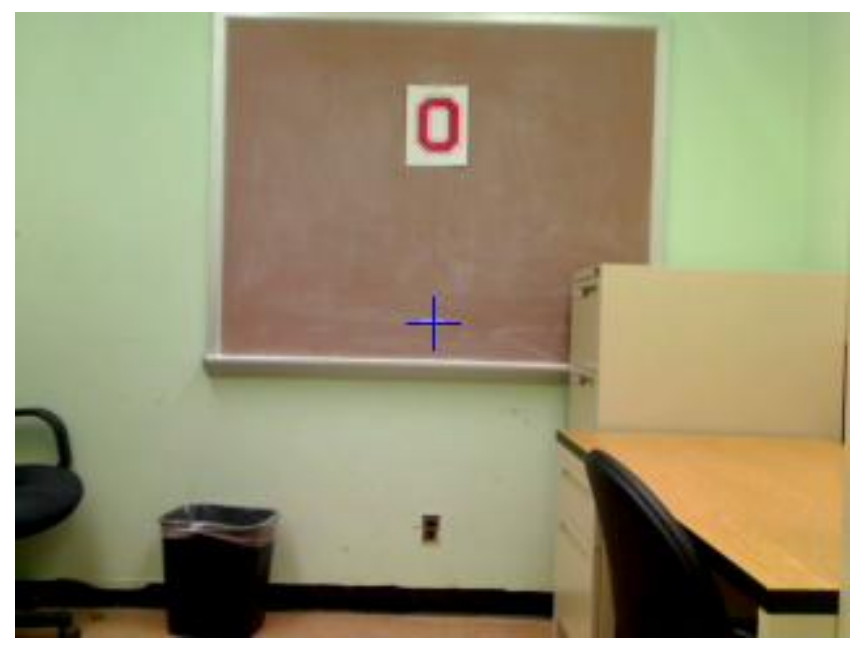

Figure 10. Method 2 Test 2

Two more experiments using this method were done but the height of the target was changed and the position of the camera was adjusted. These additional experiments can be seen in Fig. 11 and Fig. 12. The height of the target in this setup was measured to be 36 inches. Using the same approach the distances for the third test to the optical 
center and to the target were calculated to be 93.31 inches and 100.02 inches

respectively. The camera's position was moved back for the fourth test, and the distances to the optical center and the target were calculated to be 122.43 inches and 127.61 inches respectively.

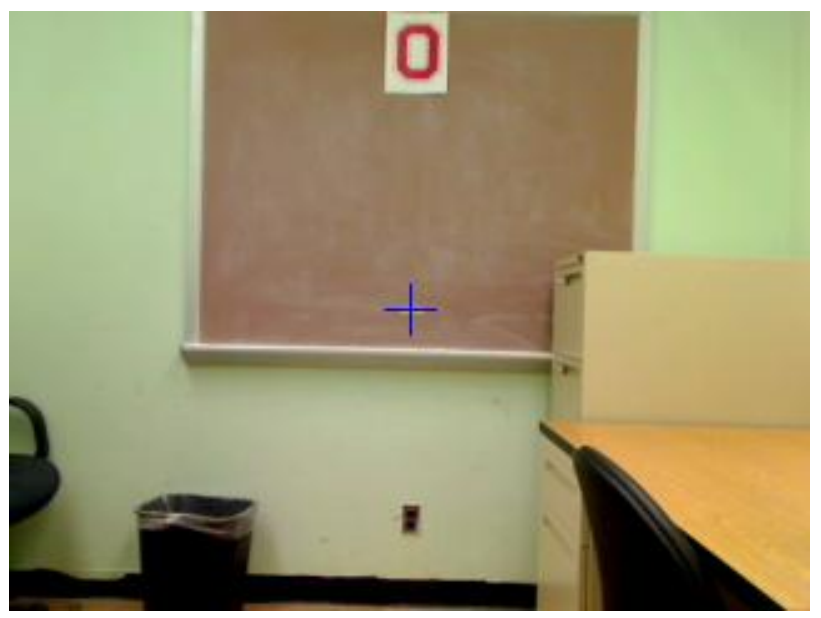

Figure 11. Method 2 Test 3

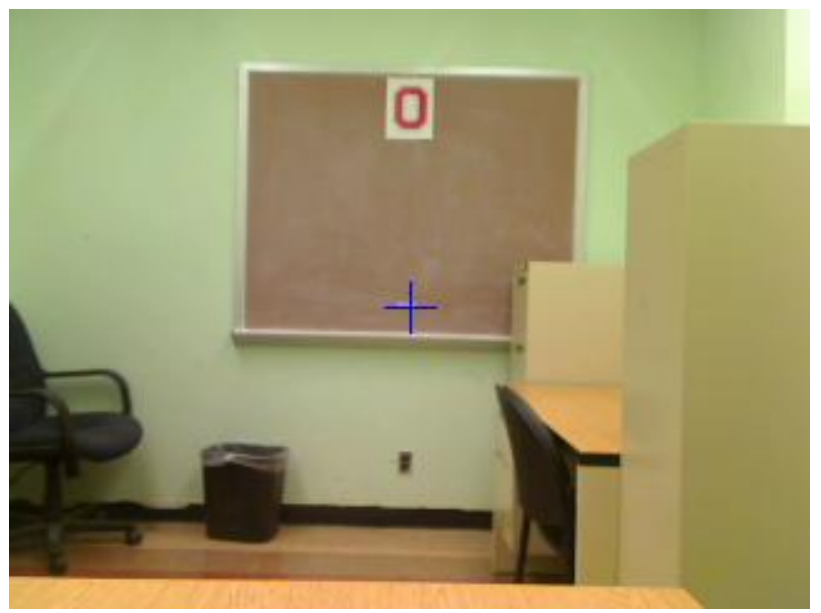

Figure 12. Method 2 Test 4 


\subsection{Method 3 Experiments}

The third method to calculate the distance to a target involves being able to calculate the angle to the target, move straight toward the target a known distance, and then calculate the angle to the target a second time. Once again, the constraint on the setup is the target must be directly above the optical center of the camera. To perform this experiment, images were taken from two locations along a straight line to the target. The images can be seen in Fig. 13 and 14. As shown in the figures, Fig. 13 is further from the target than Fig. 14, and the distance moved forward was 11.625 inches. The angles to the target were found in the same manner as done in Method 2. The angle to the target in Fig. 13 was determined to be $9.83^{\circ}$ and the angle to the target in Fig. 14 was determined to be $11.47^{\circ}$.

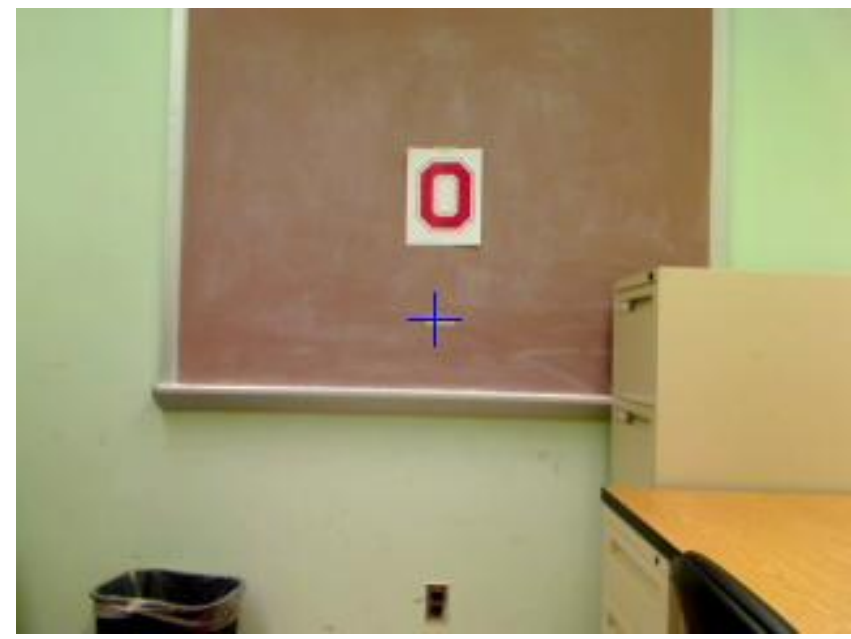

Figure 13. Method 3 Test 1 Part A 


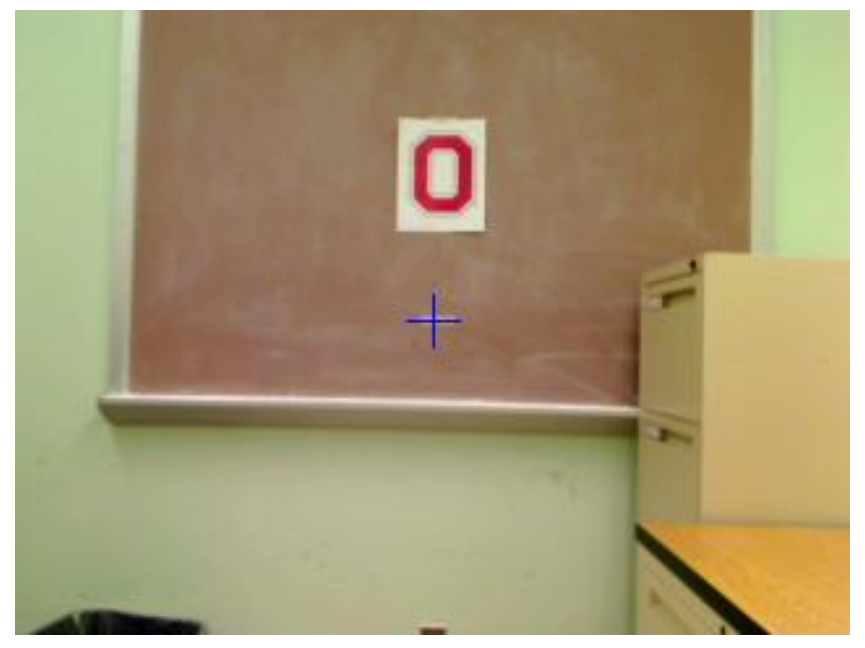

Figure 14 Method 3 Test 1 Part B

A triangle was constructed of what was known and what needed to be calculated as seen in Fig. 3. $\alpha$ was $9.83^{\circ}$ and $\beta$ was $11.47^{\circ}$ and $d$ was 11.625 inches. The distance to the optical center from the second image can be calculated by

$$
\text { distance }=\left(\frac{\sin (\alpha)}{\sin (\beta-\alpha)}\right) \cdot d \cdot \cos (\beta)
$$

The distance to the optical center from the second image was calculated to be 68.03 inches. Also, the distance to the target can be calculated by

$$
\text { distance }=\left(\frac{\sin (\alpha)}{\sin (\beta-\alpha)}\right) \cdot d
$$

The distance to the target was discovered to be 69.42 inches.

Another experiment was performed using method 3 to calculate the distance to the target. The two images for this experiment can be seen in Fig. 15 and Fig. 16. Once again, Fig. 15 is further from the target than Fig. 16. The angle to the target in Fig. 15 was determined to be $13.52^{\circ}$ and the angle to the target in Fig. 16 was determined to be 
$18.64^{\circ}$. The distance moved between the two images was 24 inches. The distance to the optical center was calculated to be 59.57 inches and the distance to the target was found to be 62.87 inches from the position in Fig. 16.

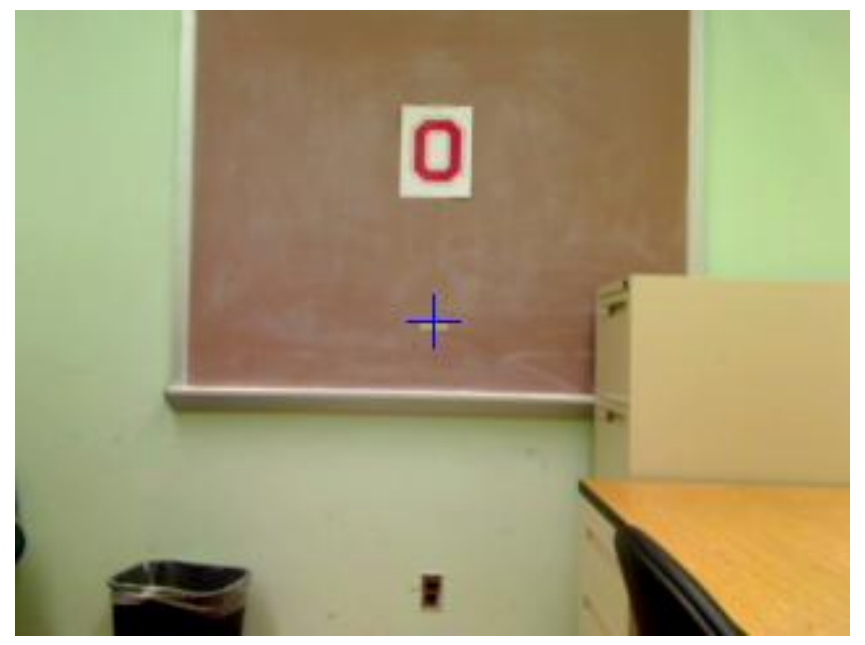

Figure 15. Method 3 Test 2 Part A

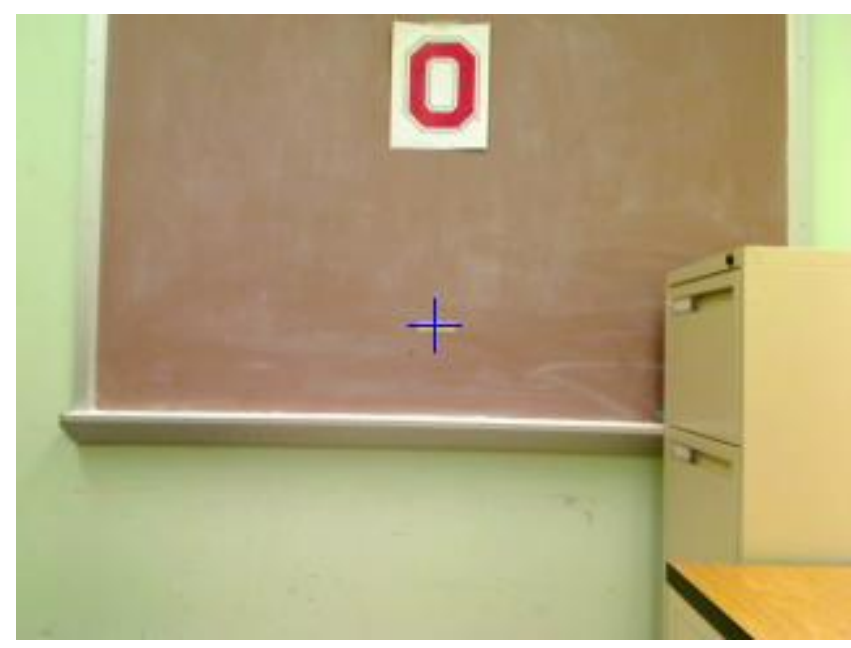

Figure 16. Method 3 Test 2 Part B 


\subsection{Method 4 Experiments}

The fourth method involves being able to see a target in the field of view of the camera. Then, being able to calculate the angle to the target in both the $\mathrm{x}$ and $\mathrm{y}$ directions. The next step would be moving forward in any direction and once again finding the angle in both directions to the target. For this experiment two images were taken using one target in the field of view, as shown in Fig. 17 and 18. However, these images differ from images from Method 3 because the target is no longer directly above the optical center of the camera.

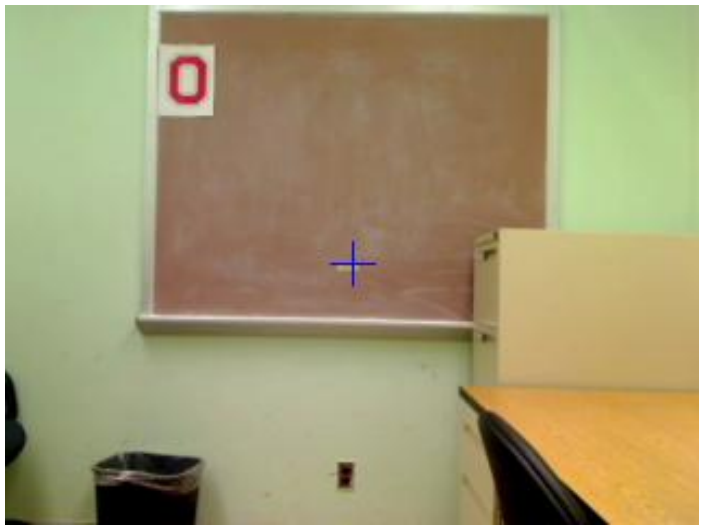

Figure 17. Method 4 Test 1 Part A

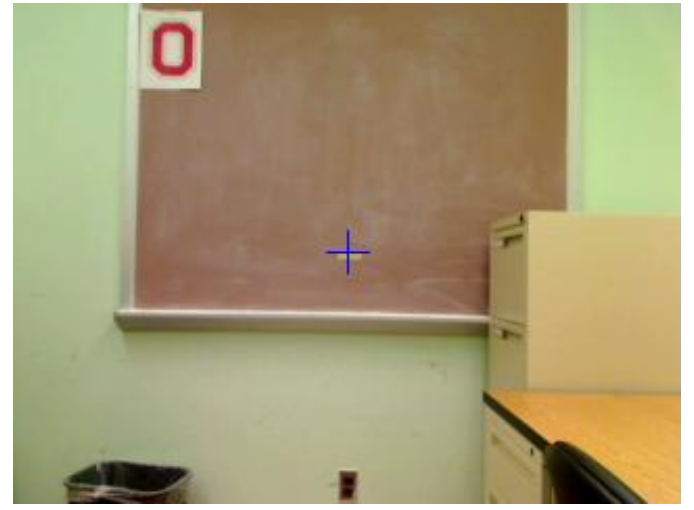

Figure 18. Method 4 Test 1 Part B 
As depicted in the images, Fig. 17 is further from the target than Fig. 18. The distance moved by the camera was 11.5 inches in some direction towards the target. In this case, the direction moved was straight toward the optical center the camera. The first step was to find the number of pixels between the optical center and the target. The angle in the $\mathrm{x}$ direction to the target was calculated based on the number of pixels between the optical center and the target, which was determined to be $14.76^{\circ}$. Next, the angle in the $y$ direction was calculated to be $17.61^{\circ}$ following the same procedure. Then, the angles in the second figure were calculated the same way, and were found to be in the $\mathrm{x}$ direction $16.73^{\circ}$ and $20.07^{\circ}$ in the y direction. The same approach that was used in Method 3 can be used to find the distance in the $\mathrm{x}$ direction to where the target, if moved down, would meet the horizon line. This same method can also be used to find the distance in the $y$ direction to where the target would meet if it were moved above the optical center. The distance along the $\mathrm{x}$ axis was calculated to be 85.32 inches and along the $\mathrm{y}$ axis was 81.15 inches. However, this only gives the distance along the optical center of the camera. In order to find the distance to the target above the center axes of the camera another distance had to be determined. From the distances calculated the height of the target can be found by using a right triangle with the information from the y direction.

$$
\text { height }=81.15 \text { inches } \cdot \sin \left(20.07^{\circ}\right)=27.85 \text { inches }
$$

Since the height of the target is known, another right triangle using the distance to the target in the $\mathrm{x}$ direction and the height of the target can be constructed to determine the distance to the target. 


$$
\text { distance }=\sqrt{27.85^{2}+85.32^{2}}=89.75 \text { inches }
$$

Another experiment was setup to test method 4 to calculate the distance to the target. The images associated with this experiment are seen in Fig. 19 and Fig. 20. The distance moved between the two images was 25.5 inches in a straight line toward the optical center. The same steps were used to calculate the height of the target from the optical center, and it was discovered to be 33.72 inches. The distance along the $\mathrm{x}$ axis was 92.35 inches, and therefore the distance to the target was 98.3157 inches.

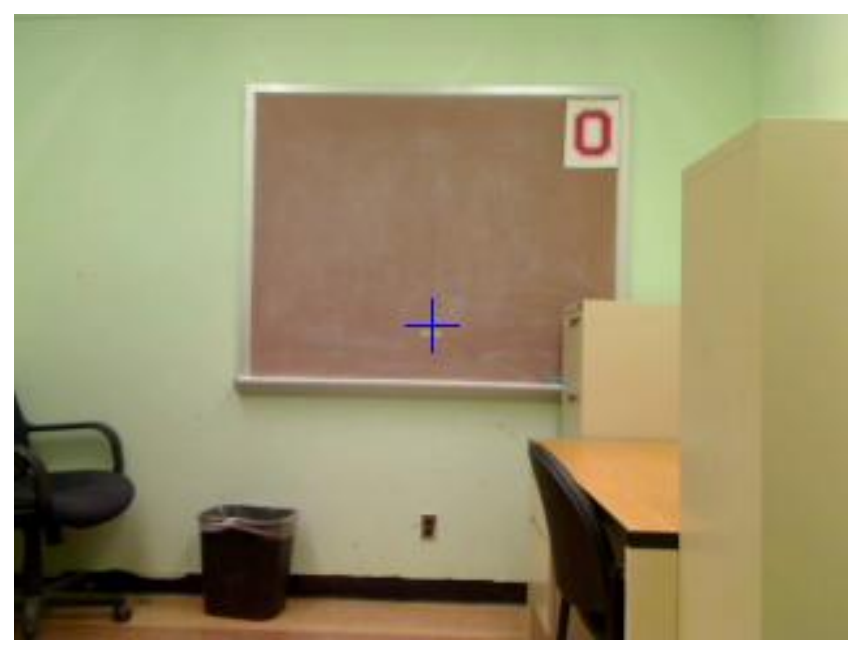

Figure 19. Method 4 Test 2 Part A 


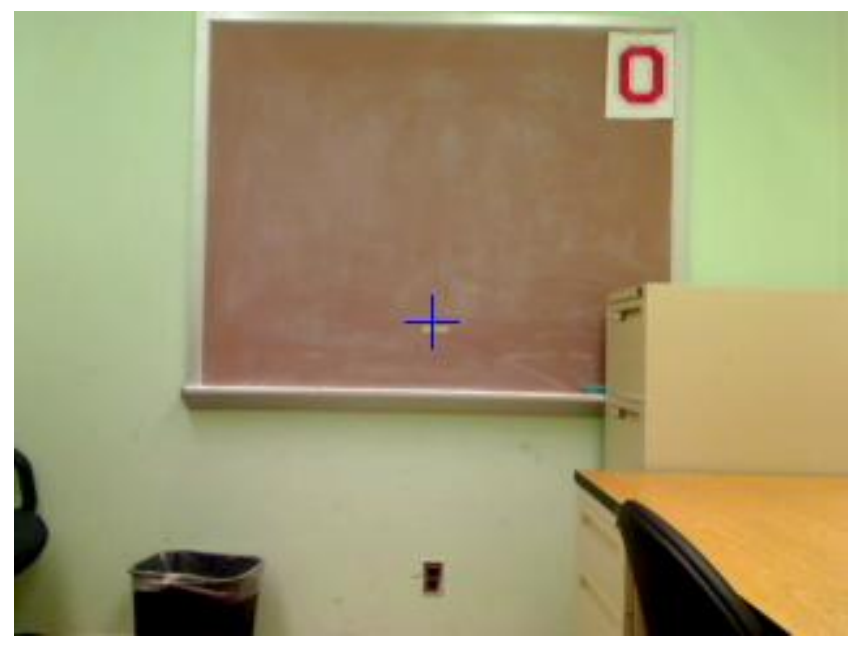

Figure 20. Method 4 Test 2 Part B

\subsection{Object Extraction and Automatic Distance Calculation}

The final step in the experimental process was to streamline the distance calculation based on size. This was done using the OpenCV software and Microsoft Visual $\mathrm{C}++$ to write a program that would detect the target and calculate the distance automatically. The first step in this process was to detect the object. The second step was to calculate the distance based on the field of view of the camera and the results given from the detection algorithm. The last step was to track the object and continue to update the distance to the object.

The first step was to detect the object. For all the experiments, a block-O object was used for the distance calculations. A color model of the block-O was formulated. The color model consisted of the white and red. The RGB values for white should all be 255, however due to lighting conditions and the paper not being completely white the thresholds for detecting white were set at RGB values to be greater than 180 . The RGB 
values for red should be $\mathrm{R}$ equal to 255 , $\mathrm{G}$ equal to 0 , and $\mathrm{B}$ equal to 0 . However, the red on the paper is not pure red and it contains green and blue in the color model, but red is still the dominant color. Therefore, the threshold for detecting red was set for $\mathrm{R}$ greater than $160, \mathrm{G}$ less than 100 , and B less than 100 .

In order to detect the object, the algorithm searched for the color white in the entire frame. Once white was found in the frame, the algorithm searched the same distance from that point in both the positive and negative horizontal directions and tried to detect the color red. When the algorithm found white in the middle of red, the block-O was detected. The next step was to detect the edges of the paper. By moving out horizontally from the detected red color, the algorithm searched for the start of white again, signifying the edge of the block-O. The algorithm then continued to move out horizontally until it no longer found the white color. Once the algorithm did not find white, it was determined that the edge of the paper was reached. When the edges were detected the object was found and the algorithm was complete.

The next step was to calculate the distance based on the camera calibration and the results of the object detection algorithm. First, the horizontal field of view was set as a variable in the distance algorithm. Next, the pixel values for the left edge and the right edge of the paper were passed to the distance algorithm from the detection program. The difference between the pixel values determines how many pixels the object occupies in the frame. Since, the actual object width is known to be 8.5 inches the number of inches per pixel can be determined by dividing the object width by the pixel difference. As was done in the camera calibration example and the method 1 examples a right triangle can be 
formed based on half of the frame size given in inches and half of the horizontal field of view angle. Finally, the distance can be calculated by taking half of the frame width and dividing it by the tangent of half of the field of view angle.

The final step was to track the object and continue to update the distance measurements. The object could change size from frame to frame based on whether the camera was moving towards or away from the object. To accomplish this task, the search for white continued in the entire frame and once white was detected the distance from that point to find red had to change. For example, if the camera were moving towards the object, the distance from the white to detect red would increase. Therefore, the tracking algorithm kept track of whether the camera was moving towards the object or away from the object. If the object was not found in the frame then the distance from white would either increase or decrease based on which way the camera was moving and the search would try again until the object was found. In order to continue to update the distance calculation, the new edges of the object were passed to the distance calculation algorithm.

The following are images taken using the detection and tracking algorithm. The distance was displayed on the command prompt while the program was running. In order to show the edges that the program was finding, blue lines were drawn, and a blue box was drawn to show that the red color was detected. Once again the $\mathrm{T}$ in the middle of the frame represents the optical center. This can be seen in Fig. 21 through Fig. 26. The distance calculated in Fig. 21 was 57.6 inches and the distance calculated in Fig. 22 was 48 inches. The rest of experiments show that from various distances the target can be detected. In all the experiments using this method a distance was given. On the final test 
using this method, the target was not at the optical center of the frame and the detection algorithm was able to locate the target and calculate the distance. This can be seen in Fig. 26.

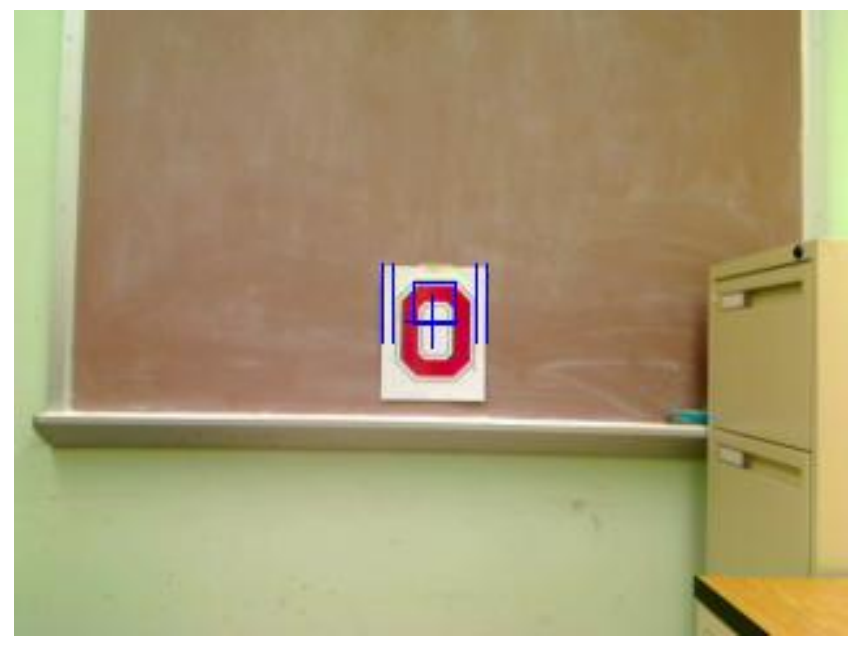

Figure 21. Automatic Detection Test 1

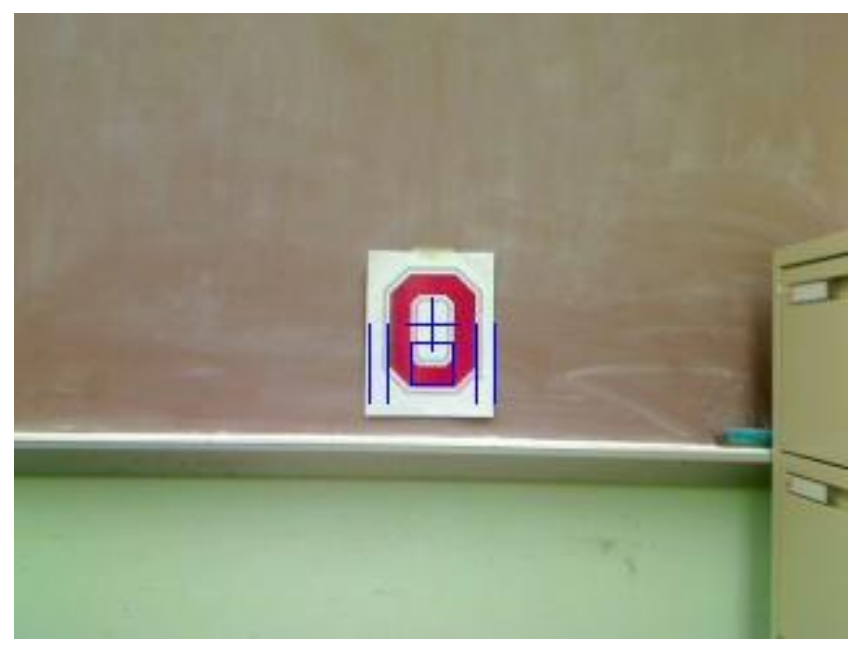

Figure 22. Automatic Detection Test 2 


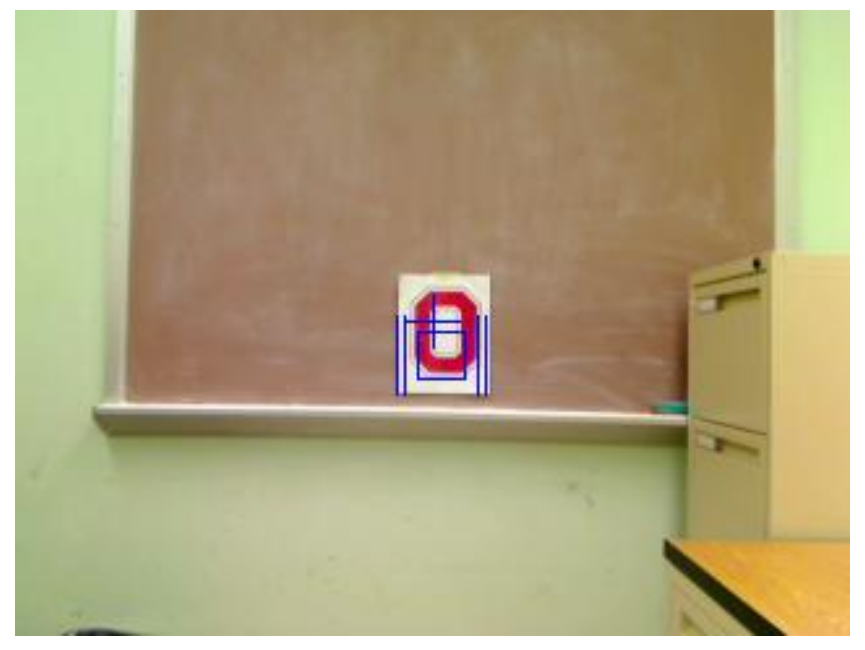

Figure 23. Automatic Detection Test 3

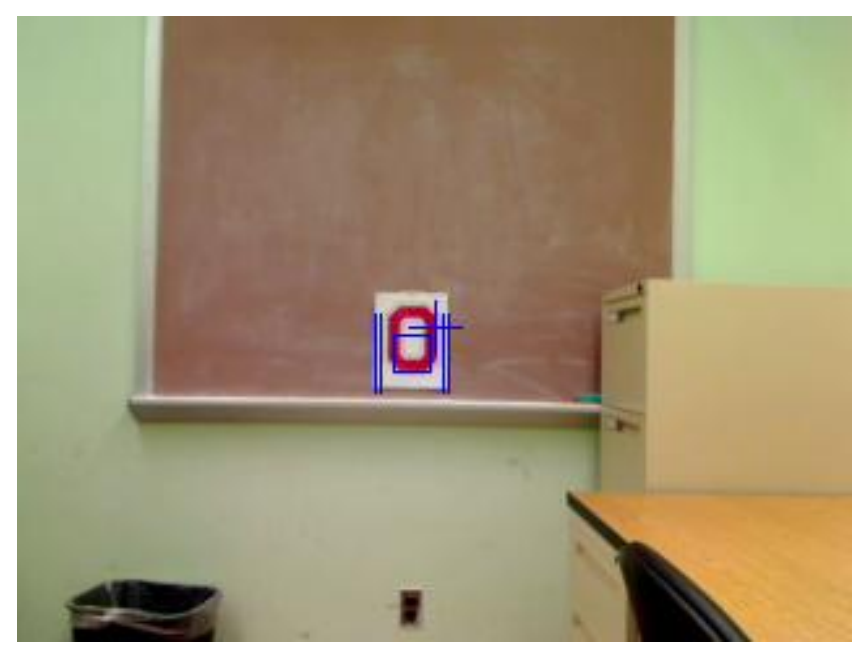

Figure 24. Automatic Detection Test 4 


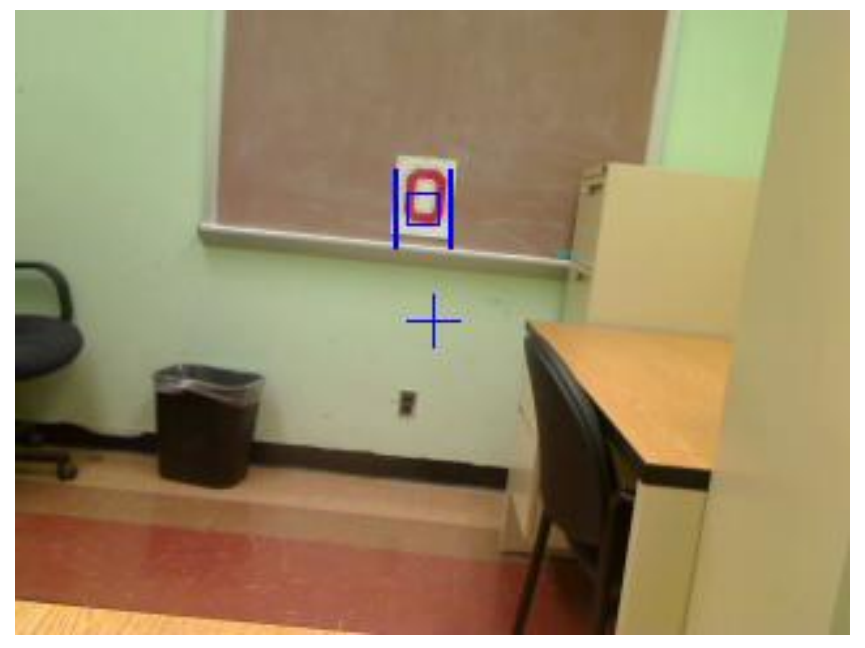

Figure 25. Automatic Detection Test 5

\subsection{Error Calculations}

For each picture taken during the experiments the distance to the target was measured using a tape measure. The actual measured distance was compared to the calculated distance and the results are tabulated in Table 1. Also shown in the table is the percent error of each measurement. The percent error is calculated using the equation

$$
\text { percent error }=\left(\frac{\mid \text { measured }- \text { calculated } \mid}{\text { measured }}\right) * 100
$$

where measured is the actual distance to the target found using the tape measure and calculated is the distance to the target found using the computer vision approach. 


\begin{tabular}{|l|c|c|c|}
\hline Test Case & $\begin{array}{c}\text { Calculated } \\
\text { Distance (Inches) }\end{array}$ & $\begin{array}{c}\text { Measured } \\
\text { Distance (Inches) }\end{array}$ & $\begin{array}{l}\text { Percent Error } \\
(\%)\end{array}$ \\
\hline M1 T1 & 59.5 & 60 & 0.833 \\
\hline M1 T2 & 79.73 & 80 & 0.3375 \\
\hline M1 T3 & 134.97 & 132 & 2.25 \\
\hline M2 T1 & 84.05 & 84 & 0.059 \\
\hline M2 T2 & 104.34 & 104.25 & 0.086 \\
\hline M2 T3 & 100.02 & 101.5 & 1.46 \\
\hline M2 T4 & 127.61 & 130.5 & 2.21 \\
\hline M3 T1 & 69.42 & 71.5 & 2.91 \\
\hline M3 T2 & 62.87 & 66.5 & 5.46 \\
\hline M4 T1 & 89.75 & 88.5 & 1.41 \\
\hline M4 T2 & 98.32 & 101.5 & 3.133 \\
\hline AD1 & 57.6 & 58.75 & 1.96 \\
\hline AD2 & 48 & 48 & 0 \\
\hline AD3 & 67.76 & 66.75 & 1.51 \\
\hline AD4 & 82.3 & 83 & 0.84 \\
\hline AD5 & 104.73 & 104.25 & 0.46 \\
\hline
\end{tabular}

Table 1. Error Calculations

From the results shown in Table 1, the Automatic Detection and Distance

Calculation provides the most accurate method of calculating the distance to the target.

All of the methods performed well calculating the distance to the target. Method 3 did not perform as well as the other methods. Method 1,2, and the automatic detection gave results with an error percentage less than 3 percent. Method 4 had a error percentage less than 4 percent, and method 3 had an error percentage of less than 6 percent. However, method 3 not performing as well could be explained by a poor setup of the images taken. For example, if the optical center does not line up with the marked center on the wall then this could cause the difference between the distances. Also, there was not much room for error when finding the edges of the targets. For example, from 132 inches, using the calculations from method 1 , a difference of 1 pixel in the $\mathrm{x}$ direction represents 8 inches in the calculated distance. From approximately 60 inches using method 1, a difference of 
1 pixel in the $\mathrm{x}$ direction represents 1.5 inches in the calculated distance. Also, all of the actual measurements were taken with a tape measure, so human error of not forcing the tape to be in a straight line or not reading the tape correctly could be cause for small discrepancies.

The error calculations show a trend in the data. As the camera moves further away from the target the error is growing. This can be seen in the results for methods 1 , 2, and 4. Using method 1 from a distance of approximately 132 inches, the error percentage was calculated to be $2.25 \%$ as compared to less than $1 \%$ for distances of 60 and 80 inches. The larger error can be explained because at larger distances each pixel represents more inches per pixel. Therefore, if the edge falls in the middle of the pixel then the calculated distance will not be as accurate as it would be for closer distances. One approach to fix this problem would be to exam the edge pixel at a sub pixel level and determine at what fraction of the pixel the edge actually occurs. Another approach would be to use a camera with a higher resolution.

The setup for all of the experiments was well done. On almost all the images the marked wall location for the optical center lined up with the actual optical center for the camera. Also, the measured distance was carefully found by maintaining a straight line between the target and the camera using the tape measure. 


\section{CHAPTER 4}

\section{CONCLUSIONS}

\subsection{Accomplishments}

The described computer vision methods complement a pseudo-satellite approach to navigation by acting as additional satellites in situations where signals are lost. This document has described four methods of finding the distance to a known target using a webcam and how to localize the platform for each method. The first step in the process to calculate distance is calibrating the camera. Once the horizontal and vertical field of view is known, the arc length per pixel can be determined in both directions. Each of the four methods used the results from the camera calibration to determine the distance to the object.

The first method uses the known size of the object to compute the distance to the object. It also uses the fact that the object is on the horizon or optical center of the camera. The second method uses the known height of the object compared to the optical center of the camera to compute the distance. Also, the object is known to be directly above the optical center of the camera. An angle from the camera to the center of the object can be determined and a right triangle formed to find the distance to the object. 
The third method uses movement combined with angle measurements to discover the height and distance to the object. The object must be directly above the optical center and the distance moved must be known in order to calculate the distance. The fourth method of calculating distance would be used in situations where direct movement towards the target cannot be achieved. In order to calculate the distance, the third method is expanded to calculate both distances in the $\mathrm{x}$ and $\mathrm{y}$ direction, and then forming a right triangle to calculate the distance to the target. This method uses the vertical and horizontal arc length of each pixel to calculate the distance. In each of the methods localization can be achieved by knowing that the target is in the field of view of the camera. Therefore, the position of the platform is constrained to being in front of the camera, by whatever distance is calculated. Since the location of the target is known, the location of the platform is also known based on the distance calculation.

Experiments using the four methods to calculate distance were performed. The experiments were set up according to the constraints of each method. The actual distance to the target was also measured for each experiment to determine how accurate each method was performing. Also, a computer program that could automatically detect the object and calculate the distance was written using the OpenCV software in Microsoft Visual $\mathrm{C}++$. This program detects the target based on the color features and shape of the block-O. The program first tries to detect a white color in the center of the $\mathrm{O}$ and then moves away from the center to detect a red color. The program also had the ability to calculate the distance to the target based on the camera calibration parameters. Experiments were run on the program and it proved to work in the setup environment. 
The error results were tabulated, and methods using manual calculations provided error rates of less than 6 percent. Automatic detection with automatic distance calculations provided error rates of less than 3 percent. The target detection algorithm worked just as well as manually locating the edges in a target using MATLAB.

\subsection{Applications}

The methods described to calculate the distance to an object using a camera can be applied to navigation. A GPS receiver may not have all four satellite signals available to calculate its position. Urban areas or areas with high foliage can cause a GPS receiver to not have access to the necessary satellites. Especially in urban areas, a target could be found that can localize the receiver by finding the distance to that target. So the camera acts as a pseudo-satellite to acquire an additional distance to localize the position of the receiver. Also, the camera can localize itself by finding the distance to an object with a known location. If the object is two dimensional, such as a sheet of paper, then the distance calculation and knowing where the object is in the field of view of the camera, the platform can localize itself with respect to the object. This application could be directly applied to indoor navigation. By placing a webcam on a mobile robot and placing targets around the environment, the robot could navigate its way around the environment using the distance calculations. The distance calculations could also be applied to persons with disabilities using wheelchairs. In nursing homes, patients that use wheelchairs, but cannot control them by themselves could use this application to navigate their way through the nursing home. Inserting a user interface into the program would allow a nurse to plan a route for the patient within the nursing home. This could be done 
by calculating the distances and when the distance to a target is within a certain limit the wheelchair must turn. After the wheelchair turns it finds the next target and goes towards it until a distance is reached. This could be repeated until the destination is achieved.

\subsection{Future Work}

The first computer vision technique of calculating the distance knowing the size of the target has already been automated in a program. The next step would be to build a program that would perform all four methods of calculating the distance. This would involve being able to detect the object from any distance, and being able to determine which method of calculating the distance needs to be performed.

Integrating the described computer vision techniques with a smart wheelchair would be a great step. The smart wheelchair has the ability to follow a wall and detect if objects are in its path by using IR sensors. However, it cannot guide itself in an indoor environment. The software that accompanies the wheelchair could be modified to include a webcam interface that would be able to track objects and calculate the distance to each object. 


\section{REFERENCES}

[1] K. Bagdonas and K. Borre. "Ubiquitous Wifi/GNSS Positioning System - TOA Based Distance Estimation," Proceeding of ION GNSS $21^{\text {st }}$ International Technical Meeting of the Satellite Division, pp. 1773-1779, September 2008.

[2] A. Brown and J. Nordlie, "Integrated GPS/TOA Navigation Using a Positioning and Communication Software Defined Radio," Proceeding of PLANS, pp. 147-152, April 2006.

[3] Y. F. Zheng, "A Pseudo-Satellite Navigation Approach Using GPS and Wavelet Range Measurement for Robust Navigation”. Unpublished report, Electrical and Computer Engineering, The Ohio State University, January, 2009.

[4] C. Peersman, S. Cvetkovic, P. Griffiths, and H. Spear. "The Global System For Mobile Communications Short Message Service," IEEE Transactions on Personal Communications, Volume 7, No. 3, pp. 15-23, June 2000.

[5] The Navigation Center of Excellence, "NAVSTAR GPS User Equipment Introduction," The Navigation Center of Excellence, Sept. 1996. [Online]. Available: http://www.navcen.uscg.gov/pubs/gps/gpsuser/gpsuser.pdf. [Accessed: March 16, 2009].

[6] D. Forsyth and J. Ponce. Computer Vision A Modern Approach. Pearson Education, Inc. Upper Saddle River, NJ, 2003. 
[7] J. Park, W. Rasheed and J. Beak. "Robot Navigation Using Camera by Identifying Arrow Signs," Proceedings of the $3^{\text {rd }}$ International Conference on Grid and Pervasive Computing - Workshops, pp. 382-386, May 2008.

[8] J. Kim and H. Jun. "Vision-Based Location Positioning using Augmented Reality for Indoor Navigation," IEEE Transactions on Consumer Electronics, Volume 54, No. 3, pp. 954-962, August 2008.

[9] C. Hsu, M. Lu and K. Chin. "Distance Measurement Based on Pixel Variation of CCD Images," Proceedings of the $4^{\text {th }}$ International Conference on Autonomous Robots and Agents, pp. 324-329, Feb., 2009.

[10] J. Song, M. Cai and M. R. Lyu. "Edge Color Distribution Transform: An Efficient Tool for Object Detection in Images," Proceeding of the $16^{\text {th }}$ International Conference on Pattern Recognition, Volume 1, pp. 608-611, Aug. 2002.

[11] S. Sheraizin and S. Itzikowitz. "Unmanned Object Detection for Image Surveillance Systems," Proceedings of IEEE EUROCON, pp. 302-307, May 2009. 